\title{
Rich gauge structures from an unitary approach of some massless gauge fields of spins one and two
}

\author{
Eugen-Mihaita CIOROIANU \\ Department of Physics, University of Craiova \\ 13 Al. I. Cuza Str., Craiova 200585, Romania \\ manache@central.ucv.ro
}

April 12, 2018

\begin{abstract}
Considering a bosonic (1-)form-valued $k$-form with a second-order Lagrangian dynamics [depending on two arbitrary real constants] we firstly perform the Dirac analysis. The procedure implies a partition of cardinally seven for the plane of the real parameters that label the starting Lagrangian. In each of the seven partition's components one determines the number and the nature of independent degrees of freedom and also a generating set of gauge transformations. Secondly, with the help of some auxiliary gauge/matter tensor gauge fields, in each of the seven situations, we construct the first-order Lagrangian density corresponding to the second-order one.
\end{abstract}

\section{Introduction}

The main building blocks of fundamental interactions consist in particles of spins one and two. These play the role of quanta for the massless gauge fields of spins one and two that mediate all the fundamental interactions in Nature. Due to this status of the spin-1 and spin-2 gauge fields, there appear the natural questions: i) is it possible to treat them in an unifying manner? and ii) if the answer is positive what benefits brings this unification? This problem was the basic clue in an old attempt to unify gravity with electromagnetism [in four spacetime dimensions] proposed by Einstein and developed by himself [1] and others [2], 3]. In the present paper we shall prove that the answer to the first question is alway positive in any spacetime dimension [greater or equal to four] at the level of free fields. In order to do this, we consider a (1-)form-valued $k$-form [ $k>2$, the values $k=1$ and $k=2$ were previously analyzed [4] that 'lives' in a $D$-dimensional Minkowski spacetime. The coefficients of this ingredient constitute the components of a tensor gauge field of degree $(k+1)$ that transform under a reducible representation of the Lorentz group whose irreducible decomposition involves two Young diagrams with one and respectively two columns. This way geometrically put on the same foot the tensor gauge fields of spin-1 [that transforms under irreducible representations of Lorentz group pictured by one-column Young diagrams [5], [6] and the tensor gauge fields of spin-2 [that pertain to the linear representations spaces of irreducible representations of the Lorentz group encoded in Young diagrams with two columns [6]. In view of dynamically similarly behaviours for the 'irreducible' components of our basic object, we consider for this a PT-invariant, second-order Lagrangian action that is labeled by two arbitrary real constants and that reduces [for particular choices of the just mentioned real parameters] to the standard Lagrangian actions for the Abelian $(k+1)$-form [5] and that of a tensor gauge field with the mixed symmetry $(k, 1)[6$. The benefits of such a unification come with the rich gauge structure displayed by the Lagrangian theory aforementioned and mainly consist in possible 'exotic' consistent interactions that can be added among one $(k+1)$-form and one tensor gauge field with the mixed symmetry $(k, 1)$.

This paper is organized into five sections as follows. In Section 2, we start with a (1-)form-valued $k$-form and interpret it as a collection of $k$-forms with a vector index. Then, we construct the most general PTinvariant and second-order Lagrangian density that is invariant under the standard gauge transformations of the just mentioned $k$-forms. The local function depends on two arbitrary real constants [denoted by $a_{1}$ and 
$a_{2}$ ] and, for some values of the real $a$-parameters, reduces to standard Lagrangian densities corresponding to the Abelian $(k+1)$-form [ $[5$ and that of a tensor gauge field with the mixed symmetry $(k, 1)[\underline{6}$. At this stage, the natural question appears: does the initial set of gauge transformations constitute a generating one? It is the job of Section 3 to prove that the answer to this question is mostly negative. Here, we perform the canonical analysis [7, [8], 9] of the starting Lagrangian theory. The procedure put into light a partition of the real parameters plane $\left(a_{1}, a_{2}\right)$ made by seven components. For six among the seven partition's components it is shown the generating set of gauge transformations is richer than the original gauge transformations, including BF [10] and/or conformal-like [1] gauge transformations. Moreover, in each of the seven situations is computed the number of degrees of freedom and is investigated the presence of unphysical degrees of freedom [ghost-modes]. It is proved the ghost-modes are absent only in two of the seven partition's components namely when the Lagrangian density reduces to that of a Abelian $(k+1)$-form and respectively to that of a tensor gauge field with the mixed symmetry $(k, 1)$. These outputs generalize the previous results [12. In view of future investigations concerning the consistent interactions that can be added among one $(k+1)$-form and one tensor gauge field with the mixed symmetry $(k, 1)$ in the context of the considered (1-)form-valued $k$-form, the Section 4 deals with the first-order formulations associated with the analyzed second-order Lagrangian theory. Here, for each of the seven partition's components, we generate the first-order Lagrangian formulation. These are done with the price of adding auxiliary gauge/matter fields that make possible the linearization. Section 5 ends the paper with the main conclusions.

\section{Setting the problem}

Our main ingredient is a (1-)form-valued $k$-form

$$
\mathbf{A}=\frac{1}{k !} A_{\mu_{1} \cdots \mu_{k} \| \alpha}\left(\mathrm{d} x^{\mu_{1}} \cdots \mathrm{d} x^{\mu_{k}}\right) \otimes \mathrm{d} x^{\alpha},
$$

that 'lives' in the $D$-dimensional Minkowski space $[D>k+1]$ of 'mostly minus' signature $\left[\sigma_{\mu \nu}=\sigma^{\mu \nu}=\right.$ $\operatorname{diag}(+,-, \cdots,-)]$. Its coordinates, $A_{\mu_{1} \cdots \mu_{k} \| \alpha}$, are the components of a bosonic tensor gauge field of degree $(k+1)$ that is antisymmetric in the first $k$ Lorentz indices

$$
A_{\mu_{1} \cdots \mu_{k} \| \alpha}=\frac{1}{k !} A_{\left[\mu_{1} \cdots \mu_{k}\right] \| \alpha}
$$

and with no symmetry in respect to the last, so one can interpret (1) in terms of a collection of $k$-forms

$$
\left\{\mathbf{A}_{\alpha}: \alpha=\overline{0, D-1}\right\}, \quad \mathbf{A}_{\alpha}=\frac{1}{k !} A_{\mu_{1} \cdots \mu_{k} \| \alpha}\left(\mathrm{d} x^{\mu_{1}} \cdots \mathrm{d} x^{\mu_{k}}\right) .
$$

Based on these, we are justified to postulate for the fields $A_{\mu_{1} \cdots \mu_{k} \| \alpha}$ the gauge transformations

$$
\delta_{\epsilon} A_{\mu_{1} \cdots \mu_{k} \| \alpha}=\partial_{\left[\mu_{1}\right.} \epsilon_{\left.\mu_{2} \cdots \mu_{k}\right] \| \alpha} .
$$

In the above, the bosonic gauge parameters $\epsilon_{\mu_{1} \cdots \mu_{k-1} \| \alpha}$ are completely antisymmetric in theirs first $(k-1)$ Lorentz indices

$$
\epsilon_{\mu_{1} \cdots \mu_{k-1} \| \alpha}=\frac{1}{(k-1) !} \epsilon_{\left[\mu_{1} \cdots \mu_{k-1}\right] \| \alpha} .
$$

The notation $[\mu \ldots \nu]$ signifies full antisymmetry with respect to the indices between brackets without normalization factors [i.e. the independent terms appear only once and are not multiplied by overall numerical factors]. In terms of the starting point (1), the gauge transformations (2) can be written as

$$
\delta_{\epsilon} \mathbf{A}=\mathrm{d} \epsilon,
$$

where

$$
\epsilon=\frac{1}{(k-1) !} \epsilon_{\mu_{1} \cdots \mu_{k-1} \| \alpha}\left(\mathrm{d} x^{\mu_{1}} \cdots \mathrm{d} x^{\mu_{k-1}}\right) \otimes \mathrm{d} x^{\alpha}
$$

is the gauge parameter vector-valued $(k-1)$-form. In the gauge transformation (3) we used the notation $\mathrm{d}$ for the de Rham differential in the exterior algebra $\wedge \mathbb{M}_{D}$. At this stage, from the perspective of the linear 
representations of the Lorentz group, the tensor gauge field $A_{\mu_{1} \cdots \mu_{k} \| \alpha}$ pertains to the reducible representation space

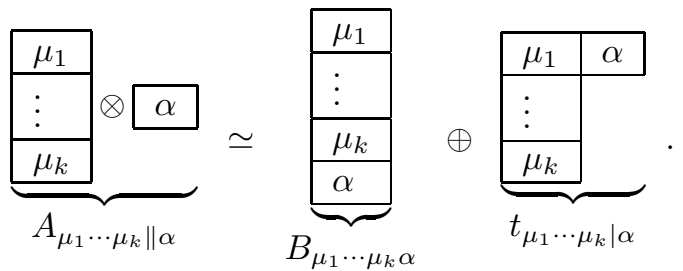

Now, we are interested in identifying the most general second-order Lagrangian density that does not break the PT-invariance and is invariant under the gauge transformations (2) [or equivalently (3i)]. In view of this, based on the gauge transformations (3), one finds the vector valued $(k+1)$-form

$$
\begin{aligned}
\mathbf{F} & \equiv \mathrm{d} \mathbf{A}=\frac{1}{(k+1) !} \partial_{\left[\mu_{1}\right.} A_{\left.\mu_{2} \cdots \mu_{k+1}\right] \| \alpha}\left(\mathrm{d} x^{\mu_{1}} \cdots \mathrm{d} x^{\mu_{k+1}}\right) \otimes \mathrm{d} x^{\alpha} \\
& \equiv \frac{1}{(k+1) !} F_{\mu_{1} \cdots \mu_{k+1} \| \alpha}\left(\mathrm{d} x^{\mu_{1}} \cdots \mathrm{d} x^{\mu_{k+1}}\right) \otimes \mathrm{d} x^{\alpha}
\end{aligned}
$$

that is manifestly gauge-invariant under (3). The seeked Lagrangian density, can be written in terms of the field-strength's coeffiecients as

$$
\mathcal{L}_{0}=\frac{1}{2(k+1)(k+1) !}\left(-\frac{(-)^{k}}{k+1}\left(F_{\mu_{1} \cdots \mu_{k+1} \| \alpha}\right)^{2}+a_{1} F_{\mu_{1} \cdots \mu_{k} \beta \| \alpha} F^{\mu_{1} \cdots \mu_{k} \alpha \| \beta}+a_{2}\left(F^{\mu_{1} \cdots \mu_{k}}\right)^{2}\right),
$$

where $a_{1}$ and $a_{2}$ are arbitrary real constants. Moreover, by $F_{\mu_{1} \cdots \mu_{k}}$ we denoted the trace of the field-strength, $F_{\mu_{1} \cdots \mu_{k}} \equiv \sigma^{\alpha \beta} F_{\mu_{1} \cdots \mu_{k} \beta \| \alpha}$ and also we employed the notation

$$
\left(U_{\Delta}\right)^{2} \equiv\left(U^{\Delta}\right)^{2} \equiv U_{\Delta} U^{\Delta}
$$

for the Lorentz multi-index $\Delta=\mu_{1} \cdots \mu_{\delta}$ contractions.

In this point, we prove that the Lagrangian density (6) can be used to treat in a unitary manner two tensor gauge fields that transform under irreducible representations of the Lorentz group, namely $(k+1)$ forms and the tensor gauge fields with the mixed symmetry $(k, 1)$. Accordingly with the isomorphysm in (4), we decompose the gauge field $A_{\mu_{1} \cdots \mu_{k} \| \alpha}$ into its 'irreducible' components

$$
A_{\mu_{1} \cdots \mu_{k} \| \alpha} \equiv B_{\mu_{1} \cdots \mu_{k} \alpha}+t_{\mu_{1} \cdots \mu_{k} \mid \alpha} \equiv\left(\frac{1}{k+1} A_{\left[\mu_{1} \cdots \mu_{k} \| \alpha\right]}\right)+\left(A_{\mu_{1} \cdots \mu_{k} \| \alpha}-\frac{1}{k+1} A_{\left[\mu_{1} \cdots \mu_{k} \| \alpha\right]}\right) .
$$

Replacing this split into the definition of the field-strength (5) we get

$$
F_{\mu_{1} \cdots \mu_{k+1} \| \alpha}=H_{\mu_{1} \cdots \mu_{k+1} \alpha}+(-)^{k} \partial_{\alpha} B_{\mu_{1} \cdots \mu_{k+1}}+\mathcal{F}_{\mu_{1} \cdots \mu_{k+1} \mid \alpha},
$$

where we employed the notations

$$
H_{\mu_{1} \cdots \mu_{k+1} \alpha} \equiv \partial_{\left[\mu_{1}\right.} B_{\left.\mu_{2} \cdots \mu_{k+1} \alpha\right]}, \quad \mathcal{F}_{\mu_{1} \cdots \mu_{k+1} \mid \alpha} \equiv \partial_{\left[\mu_{1}\right.} t_{\left.\mu_{2} \cdots \mu_{k+1}\right] \mid \alpha}
$$

for the field-strengths corresponding to the 'irreducible' components $B_{\mu_{1} \cdots \mu_{k} \alpha}$ and $t_{\mu_{1} \cdots \mu_{k} \mid \alpha}$. Based on the result (8), by direct computations, we bring the Lagrangian density (6) under the form

$$
\begin{aligned}
\mathcal{L}_{0}= & -\frac{(-)^{k}+\left(k^{2}+k+1\right) a_{1}+a_{2}}{2(k+1)^{2}(k+2) !}\left(H_{\mu_{1} \cdots \mu_{k+2}}\right)^{2}+\frac{a_{1}+a_{2}-(-)^{k}}{2(k+1)^{2}(k+1) !}\left(\partial_{\alpha} B_{\mu_{1} \cdots \mu_{k+1}}\right)^{2} \\
& +\frac{a_{1}-(-)^{k}}{2(k+1)^{2}(k+1) !}\left(\mathcal{F}_{\mu_{1} \cdots \mu_{k+1} \mid \alpha}\right)^{2}+\frac{a_{2}}{2(k+1)(k+1) !}\left(\mathcal{F}_{\mu_{1} \cdots \mu_{k}}\right)^{2} \\
& +(-)^{k} \frac{a_{1}+a_{2}-(-)^{k}}{(k+1)^{2}(k+1) !} \mathcal{F}_{\mu_{1} \cdots \mu_{k+1} \mid \alpha} \partial^{\alpha} B^{\mu_{1} \cdots \mu_{k+1}}+\partial_{\mu} j^{\mu} .
\end{aligned}
$$

Here $\mathcal{F}_{\mu_{1} \cdots \mu_{k}}$ is nothing but the trace of the tensor $\mathcal{F}_{\mu_{1} \cdots \mu_{k+1} \mid \alpha}\left[\mathcal{F}_{\mu_{1} \cdots \mu_{k}} \equiv \sigma^{\alpha \beta} \mathcal{F}_{\mu_{1} \cdots \mu_{k} \alpha \mid \beta}\right]$. Also, the components of the local current in the left hand side of the density (8) have the concrete expressions

$$
\begin{aligned}
j^{\mu}= & \frac{a_{2}}{2(k+1)(k+1) !}\left(B^{\mu \mu_{1} \cdots \mu_{k}} \partial^{\rho} B_{\rho \mu_{1} \cdots \mu_{k}}-B_{\rho \mu_{1} \cdots \mu_{k}} \partial^{\rho} B^{\mu \mu_{1} \cdots \mu_{k}}\right. \\
& \left.+2 B^{\mu \mu_{1} \cdots \mu_{k}} \mathcal{F}_{\mu_{1} \cdots \mu_{k}}-\frac{2(-)^{k}}{k+1} B_{\mu_{1} \cdots \mu_{k+1}} \mathcal{F}^{\mu_{1} \cdots \mu_{k+1} \mid \mu}\right) .
\end{aligned}
$$


The Lagrangian action based on the local function (10)

$$
\begin{aligned}
& S_{0}^{\mathrm{L}}\left[B_{\mu_{1} \cdots \mu_{k+1}}, t_{\mu_{1} \cdots \mu_{k} \mid \alpha}\right]=\int \mathrm{d}^{D} x\left[(-)^{k} \frac{a_{1}+a_{2}-(-)^{k}}{(k+1)^{2}(k+1) !} \mathcal{F}_{\mu_{1} \cdots \mu_{k+1} \mid \alpha} \partial^{\alpha} B^{\mu_{1} \cdots \mu_{k+1}}\right. \\
& -\frac{(-)^{k} k+\left(k^{2}+k+1\right) a_{1}+a_{2}}{2(k+1)^{2}(k+2) !}\left(H_{\mu_{1} \cdots \mu_{k+2}}\right)^{2}+\frac{a_{1}+a_{2}-(-)^{k}}{2(k+1)^{2}(k+1) !}\left(\partial_{\alpha} B_{\mu_{1} \cdots \mu_{k+1}}\right) \partial^{\alpha} B^{\mu_{1} \cdots \mu_{k+1}} \\
& \left.+\frac{a_{1}-(-)^{k}}{2(k+1)^{2}(k+1) !}\left(\mathcal{F}_{\mu_{1} \cdots \mu_{k+1} \mid \alpha}\right)^{2}+\frac{a_{2}}{2(k+1)(k+1) !}\left(\mathcal{F}_{\mu_{1} \cdots \mu_{k}}\right)^{2}\right]
\end{aligned}
$$

governs the dynamics of the tensor fields $t_{\mu_{1} \cdots \mu_{k} \mid \alpha}$ and $B_{\mu_{1} \cdots \mu_{k} \alpha}$ that transform under irreducible representations of the Lorentz group. In (12) the components $t_{\mu_{1} \cdots \mu_{k} \mid \alpha}$ and $B_{\mu_{1} \cdots \mu_{k} \alpha}$ do not mix iff the constants $a_{1}$ and $a_{2}$ are subjects to the algebraic equation

$$
a_{1}+a_{2}-(-)^{k}=0 \Leftrightarrow a_{2}=(-)^{k}-a_{1} .
$$

Indeed, by inserting the solution (13) in the right hand side of (12) we get

$$
S_{0}^{\mathrm{L}}\left[B_{\mu_{1} \cdots \mu_{k+1}}, t_{\mu_{1} \cdots \mu_{k} \mid \alpha}\right]=\frac{(-)^{k}+k a_{1}}{k+1} S_{0}^{(\mathrm{k}+1)}\left[B_{\mu_{1} \cdots \mu_{k+1}}\right]+\frac{a_{1}-(-)^{k}}{(k+1)^{2}} S_{0}^{(\mathrm{k}, 1)}\left[t_{\mu_{1} \cdots \mu_{k} \mid \alpha}\right],
$$

where $S_{0}^{(\mathrm{k}+1)}\left[B_{\mu_{1} \cdots \mu_{k+1}}\right]$ and $S_{0}^{(\mathrm{k}, 1)}\left[t_{\mu_{1} \cdots \mu_{k} \mid \alpha}\right]$ are the standard actions for $(k+1)$-form [5] and respectively for the massless tensor gauge field with the mixed symmetry $(k, 1)[6]$. Now, if we set in (14)

$$
a_{1}=(-)^{k},
$$

the $(k+1)$-form $B_{\mu_{1} \cdots \mu_{k+1}}$ becomes a pure gauge field. Also, tacking in (14)

$$
a_{1}=-\frac{(-)^{k}}{k}
$$

the field $t_{\mu_{1} \cdots \mu_{k} \mid \alpha}$ with the mixed symmetry $(k, 1)$ becomes a pure gauge one.

The above analysis allows us to conclude that some tensor gauge fields of degree $(k+1)$ that transform under irreducible representations of the Lorentz group can be treated in unified manner through the gauge field $A_{\mu_{1} \cdots \mu_{k} \| \alpha}$ whose dynamics is generated by the Lagrangian density ([6).

\section{Dirac analysis}

In this section we perform the canonical analysis [7], 8], [9] of the model with the Lagrangian density (6). In view of this, if we denote by $\pi_{\mu_{1} \cdots \mu_{k} \| \alpha}$ the canonical momenta associated with the fields $A^{\mu_{1} \cdots \mu_{k} \| \alpha}$, the definitions of the formers read as

$$
\begin{aligned}
\pi_{\mu_{1} \cdots \mu_{k} \| \alpha} & \equiv \frac{1}{k !} \frac{\partial \mathcal{L}_{0}}{\partial \dot{\dot{A}}^{\left[\mu_{1} \cdots \mu_{k}\right] \| \alpha}} \\
& =\frac{(-)^{k+1}}{(k+1)(k+1) !}\left(F_{0 \mu_{1} \cdots \mu_{k} \| \alpha}-a_{1} F_{\alpha\left[0 \mu_{1} \cdots \mu_{k-1} \| \mu_{k}\right]}-a_{2} \sigma_{\alpha[0} F_{\left.\mu_{1} \cdots \mu_{k}\right]}\right)
\end{aligned}
$$

where by overdot we denoted the derivative in respect with the temporal coordinate $x^{0}$. From the definitions in the above, we infer the primary constraints

$$
G_{i_{1} \cdots i_{k-1}}^{(1)} \equiv \pi_{0 i_{1} \cdots i_{k-1} \| 0} \approx 0, \quad G_{i_{1} \cdots i_{k-1} \| j}^{(1)} \equiv \pi_{0 i_{1} \cdots i_{k-1} \| j} \approx 0
$$

and also the relations

$$
\begin{aligned}
& \pi_{i_{1} \cdots i_{k} \| 0}=\frac{1}{(k+1)(k+1) !}\left[\left(a_{1}+a_{2}-(-)^{k}\right) F_{0 i_{1} \cdots i_{k} \| 0}+(-)^{k} a_{2} F_{i_{1} \cdots i_{k}}^{\prime}\right], \\
& \pi_{i_{1} \cdots i_{k} \| j}=\frac{(-)^{k+1}}{(k+1)(k+1) !}\left(F_{0 i_{1} \cdots i_{k} \| j}-a_{1} F_{j\left[0 i_{1} \cdots i_{k-1} \| i_{k}\right]}-(-)^{k} a_{2} \sigma_{j\left[i_{1}\right.} F_{\left.i_{2} \cdots i_{k}\right] 0}\right) .
\end{aligned}
$$


In formula (19) we denoted by $F_{i_{1} \cdots i_{k}}^{\prime}$ the spatial part of the field-strength's trace $\left[F_{i_{1} \cdots i_{k}}^{\prime} \equiv \sigma^{j l} F_{i_{1} \cdots i_{k} j \| l}\right]$. In the flow of the analysis we will also use the 'irreducible' components of the primary constraints (18)

$$
\begin{aligned}
G_{i_{1} \cdots i_{k-1} \| j}^{(1)} & \equiv\left(\frac{1}{k} \pi_{0\left[i_{1} \cdots i_{k-1} \| j\right]}\right)+\left(\pi_{0 i_{1} \cdots i_{k-1} \| j}-\frac{1}{k} \pi_{0\left[i_{1} \cdots i_{k-1} \| j\right]}\right) \\
& \equiv\left(\frac{1}{k} G_{\left[i_{1} \cdots i_{k-1} \| j\right]}^{(1)}\right)+\left(G_{i_{1} \cdots i_{k-1} \| j}^{(1)}-\frac{1}{k} G_{\left[i_{1} \cdots i_{k-1} \| j\right]}^{(1)}\right) \equiv G_{i_{1} \cdots i_{k}}^{(1)}+G_{i_{1} \cdots i_{k-1} \mid j}^{(1)} .
\end{aligned}
$$

Based on the equations (20), only by algebraic computations, we derive

$$
\begin{aligned}
\pi_{i_{1} \cdots i_{k-1}}^{\prime} & \equiv \sigma^{j k} \pi_{i_{1} \cdots i_{k-1} j \| k}=\frac{a_{1}+a_{2}(D-k)-(-)^{k}}{(k+1)(k+1) !} F_{0 i_{1} \cdots i_{k-1}}, \\
\pi_{\left[i_{1} \cdots i_{k} \| i_{k+1}\right]} & =\frac{(-)^{k}}{(k+1) !}\left[a_{1} F_{i_{1} \cdots i_{k+1} \| 0}-(-)^{k} \frac{k a_{1}+(-)^{k}}{(k+1)} F_{0\left[i_{1} \cdots i_{k} \| i_{k+1}\right]}\right] .
\end{aligned}
$$

The first step in the canonical analysis is achived by solving the equations (19)-(20) in respect with some of the generalized velocities. In view of this, the results (22)-(23) lead to seven distinct situations [dictated by the factors that multiply the temporal components of the field-strength in (19)-(20)], namely

$$
\begin{aligned}
a_{1} & =(-)^{k}, \quad a_{2}=0 ; \\
a_{1} & =-\frac{(-)^{k}}{k}, \quad a_{2}=(-)^{k} \frac{k+1}{k} ; \\
a_{1} & =-\frac{(-)^{k}}{k}, \quad a_{2}=(-)^{k} \frac{k+1}{k(D-k)} ; \\
a_{1} & =-\frac{(-)^{k}}{k}, \quad a_{2} \equiv a \in \mathbb{R} \backslash\left\{(-)^{k} \frac{k+1}{k},(-)^{k} \frac{k+1}{k(D-k)}\right\} ; \\
a_{1} & \equiv \bar{a} \in \mathbb{R} \backslash\left\{-\frac{(-)^{k}}{k},(-)^{k}\right\}, \quad a_{2}=\frac{(-)^{k}-\bar{a}}{D-k} ; \\
a_{1} & \equiv \tilde{a} \in \mathbb{R} \backslash\left\{-\frac{(-)^{k}}{k},(-)^{k}\right\}, \quad a_{2}=(-)^{k}-\tilde{a} ; \\
a_{1}+a_{2} & \neq(-)^{k} \neq a_{1}+(D-k) a_{2}, \quad a_{1} \in \mathbb{R} \backslash\left\{(-)^{k}\right\} .
\end{aligned}
$$

In the remaining part of this section we will complete the canonical analysis of the model in each of the seven situations delimited in the above. This will include a careful analysis of the nature of independent degrees of freedom [physical/ghost modes].

\subsection{Case I}

In the situation (24) the Lagrangian density (6) becomes

$$
\mathcal{L}_{0}^{(I)}=\frac{(-)^{k}}{2(k+1)(k+1) !}\left(-\frac{1}{k+1} F_{\mu_{1} \cdots \mu_{k+1} \| \alpha} F^{\mu_{1} \cdots \mu_{k+1} \| \alpha}+F_{\mu_{1} \cdots \mu_{k} \beta \| \alpha} F^{\mu_{1} \cdots \mu_{k} \alpha \| \beta}\right)
$$

and the definitions of the canonical momenta (17) lead to the independent primary constraints (18) and

$$
\begin{aligned}
\gamma_{i_{1} \cdots i_{k}}^{(1)} & \equiv \pi_{i_{1} \cdots i_{k} \| 0} \approx 0, \\
\gamma_{i_{1} \cdots i_{k} \mid j}^{(1)} & \equiv \pi_{i_{1} \cdots i_{k} \| j}-\frac{1}{k+1} \pi_{\left[i_{1} \cdots i_{k} \| j\right]} \approx 0 .
\end{aligned}
$$

Solving now the equations (17) [corresponding to the choice (24) of the real parameters $a_{1}$ and $a_{2}$ ] in respect with some of the generalized velocities, we get the canonical Hamiltonian

$$
\begin{aligned}
\mathcal{H}_{0}^{(I)}= & -k A^{0 i_{1} \cdots i_{k-1} \| j} \partial^{l} \pi_{l i_{1} \cdots i_{k-1} \| j}-\frac{(-)^{k} k !}{2} \pi_{i_{1} \cdots i_{k} \| j} \pi^{\left[i_{1} \cdots i_{k} \| j\right]} \\
& +\frac{(-)^{k}}{(k+1)^{2}} \pi^{\left[i_{1} \cdots i_{k} \| j\right]} F_{i_{1} \cdots i_{k} j \| 0}+\frac{(-)^{k}}{2(k+1)(k+2) !} F_{i_{1} \cdots i_{k} \| j} F^{\left[i_{1} \cdots i_{k} \| j\right]} .
\end{aligned}
$$

As the primary constraints (18) and (32)-(33) depend only on the momenta we gather their Abelian character so that the consistency of the primary constraints reduces only to the computation of the Poisson brackets between them and the canonical Hamiltonian. In view of these, simple computations lead to

$$
\left[G_{i_{1} \cdots i_{k-1}}^{(1)}, \mathcal{H}_{0}^{(I)}\right]=0=\left[\gamma_{i_{1} \cdots i_{k} \mid j}^{(1)}, \mathcal{H}_{0}^{(I)}\right]
$$


and

$$
\begin{aligned}
{\left[\gamma_{i_{1} \cdots i_{k}}^{(1)}, \mathcal{H}_{0}^{(I)}\right] } & =\frac{1}{k+1} \partial^{j} \pi_{\left[i_{1} \cdots i_{k} \| j\right]} \equiv \gamma_{i_{1} \cdots i_{k}}^{(2)} \approx 0, \\
{\left[G_{i_{1} \cdots i_{k-1} \| j}^{(1)}, \mathcal{H}_{0}^{(I)}\right] } & =\partial^{i} \gamma_{i i_{1} \cdots i_{k} \mid j}^{(1)}+(-)^{k} \gamma_{i_{1} \cdots i_{k-1} j}^{(2)} \approx 0
\end{aligned}
$$

results that reveal the secondary constraints

$$
\gamma_{i_{1} \cdots i_{k}}^{(2)} \equiv \frac{1}{k+1} \partial^{j} \pi_{\left[i_{1} \cdots i_{k} \| j\right]} \approx 0 .
$$

Invoking again the dependence of the constraints (18), (32)-(33) and (38) only on the canonical momenta, we establish theirs Abelianity. This remark, together with the Poisson brackets

$$
\left[\gamma_{i_{1} \cdots i_{k}}^{(2)}, \mathcal{H}_{0}^{(I)}\right]=0
$$

allows us to conclude that the Dirac algorithm stops at this stage.

In order to count the independent degrees of freedom, we invoke the first-class character of the constraints set (18), (32)-(33) and (38) supplemented with the off-shell reducibilities of order $L=k$ of the secondary constraints (38)

$$
\begin{aligned}
\left(Z_{j_{1} \cdots j_{k-1}}\right)^{i_{1} \cdots i_{k}} \gamma_{i_{1} \cdots i_{k}}^{(2)} & =0 \\
\left(Z_{l_{1} \cdots l_{k-p-2}}\right)^{j_{1} \cdots j_{k-p-1}}\left(Z_{j_{1} \cdots j_{k-p-1}}\right)^{i_{1} \cdots i_{k-p}} & =0, \quad p=\overline{0, k-2} .
\end{aligned}
$$

In the above we used the notations

$$
\left(Z_{j_{1} \cdots j_{k-p-1}}\right)^{i_{1} \cdots i_{k-p}}=\partial^{\left[i_{1}\right.} \delta_{j_{1}}^{i_{2}} \cdots \delta_{j_{k-p-1}}^{\left.i_{k-p}\right]}, \quad p=\overline{0, k-1} .
$$

The arguments that we have just given allow us to conclude that: the canonical Hamiltonian (34) is of the first-class [so this is the classical observable that governs the time evolution] and the number of independent degrees of freedom for the model under study is

$$
N_{D O F}^{(I)}=\left(\begin{array}{c}
D-2 \\
k+1
\end{array}\right) .
$$

Next, we analyze the nature [physical/unphysical] of the degrees of freedom (41). In view of this, we firstly pass to the reduced phase-space [by choosing of some apropriate canonical gauge conditions]. Then, we evaluate the kinetic term of the first-class Hamiltonian (34) restricted to the reduced phase-space. If the kinetic term possesses definitness [negatively or positively] then all degrees of freedom are physical. Otherwise, ghost modes are present among the degrees of freedom.

In our case, a set of canonical gauge conditions consists in

$$
\begin{aligned}
\chi^{(1) i_{1} \cdots i_{k-1}} & \equiv A^{0 i_{1} \cdots i_{k-1} \| 0} \approx 0, \quad \chi^{(1) i_{1} \cdots i_{k-1} \| j} \equiv A^{0 i_{1} \cdots i_{k-1} \| j} \approx 0, \\
\bar{\chi}^{(1) i_{1} \cdots i_{k}} & \equiv A^{i_{1} \cdots i_{k} \| 0} \approx 0, \quad \bar{\chi}^{(1) i_{1} \cdots i_{k} \mid j} \equiv A^{i_{1} \cdots i_{k} \| j}-\frac{1}{k+1} A^{\left[i_{1} \cdots i_{k} \| j\right]} \approx 0
\end{aligned}
$$

and

$$
\bar{\chi}^{(2) i_{1} \cdots i_{k}} \equiv \frac{1}{k+1} \partial_{j} A^{\left[i_{1} \cdots i_{k} \| j\right]} \approx 0
$$

Now, looking at the restriction

$$
\overline{\mathcal{H}}_{0}^{(I)} \approx-\frac{(-)^{k} k !}{2} \pi_{i_{1} \cdots i_{k} \| j} \pi^{\left[i_{1} \cdots i_{k} \| j\right]}+\frac{(-)^{k}}{2(k+1)(k+2) !} F_{i_{1} \cdots i_{k} \| j} F^{\left[i_{1} \cdots i_{k} \| j\right]}
$$

of the first-class Hamiltonian on the reduced phase space, we conclude that all degrees of freedom (41) are physicall.

Finally, based on the Dirac's conjecture [according to which any first-class constraint generates gauge transformations], if we pass again to the Lagrangian formulation [via extended action], we derive for the functional

$$
S_{0}^{(I)}\left[A_{\mu_{1} \cdots \mu_{k} \| \alpha}\right]=\int \mathrm{d}^{D} x \mathcal{L}_{0}^{(I)}
$$


the generating set of gauge transformations

$$
\delta_{\epsilon, \xi}^{(I)} A_{\mu_{1} \cdots \mu_{k} \| \alpha}=\partial_{\left[\mu_{1}\right.} \epsilon_{\left.\mu_{2} \cdots \mu_{k} \alpha\right]}+\xi_{\mu_{1} \cdots \mu_{k} \mid \alpha}
$$

where the gauge parameters $\xi_{\mu_{1} \cdots \mu_{k} \mid \alpha}$ have the mixed symmetry $(k, 1)$

$$
\xi_{\mu_{1} \cdots \mu_{k} \mid \alpha}=\frac{1}{k !} \xi_{\left[\mu_{1} \cdots \mu_{k}\right] \mid \alpha}, \quad \xi_{\left[\mu_{1} \cdots \mu_{k} \mid \alpha\right]}=0 .
$$

We observe that the results (47) imply that the 'irreducible' component $t_{\mu_{1} \ldots \mu_{k} \mid \alpha}$ [with the concrete expression given in (7)] is a pure gauge field

$$
\delta_{\epsilon, \xi}^{(I)} t_{\mu_{1} \cdots \mu_{k} \mid \alpha}=\xi_{\mu_{1} \cdots \mu_{k} \mid \alpha}
$$

output that agrees with the discution in the end of the previous subsection.

\subsection{Case II}

Now, we complete the canonical analysis of the model (6) in the second situation [the real parameters $a_{1}$ and $a_{2}$ take the values (25)]. In this context, the Lagrangian density (6) becomes

$$
\mathcal{L}_{0}^{(I I)}=\frac{(-)^{k+1}}{2(k+1)(k+1) !}\left[\frac{1}{k+1}\left(F_{\mu_{1} \cdots \mu_{k+1} \| \alpha}\right)^{2}+\frac{1}{k} F_{\mu_{1} \cdots \mu_{k} \beta \| \alpha} F^{\mu_{1} \cdots \mu_{k} \alpha \| \beta}-\frac{k+1}{k}\left(F_{\mu_{1} \cdots \mu_{k}}\right)^{2}\right] .
$$

Based on the choice (25), the definitions of the canonical momenta (17) lead to the independent primary constraints (18) and

$$
\begin{aligned}
\bar{\gamma}_{i_{1} \cdots i_{k}}^{(1)} & \equiv \pi_{i_{1} \cdots i_{k} \| 0}-\frac{1}{k \cdot(k+1) !} F_{i_{1} \cdots i_{k}}^{\prime} \approx 0, \\
\bar{\gamma}_{i_{1} \cdots i_{k+1}}^{(1)} & \equiv \pi_{\left[i_{1} \cdots i_{k} \| i_{k+1}\right]}+\frac{1}{k \cdot(k+1) !} F_{i_{1} \cdots i_{k+1} \| 0} \approx 0 .
\end{aligned}
$$

Solving the equations (17) in respect with some of the generalized velocities, we derive the canonical Hamiltonian density [well defined only on the primary constraint surface]

$$
\begin{aligned}
\mathcal{H}_{0}^{(I I)}= & -k A^{0 i_{1} \cdots i_{k-1} \| \mu}\left(\partial^{l} \pi_{l i_{1} \cdots i_{k-1} \| \mu}\right)+\frac{(-)^{k}}{2(k+1)^{2}(k+1) !}\left(F_{i_{1} \cdots i_{k+1} \| \mu}\right)^{2} \\
& +\frac{(-)^{k}}{2 k(k+1)(k+1) !} F_{i_{1} \cdots i_{k} j \| l} F^{i_{1} \cdots i_{k} l \| j}-\frac{(-)^{k}}{2 k(k+1) !}\left(F_{i_{1} \cdots i_{k}}^{\prime}\right)^{2} \\
& -\frac{(-)^{k} k(k+1) !}{2} \pi_{i_{1} \cdots i_{k} \| j} \pi^{i_{1} \cdots i_{k} \| j}-\frac{(-)^{k}}{2(k+1)} \pi_{i_{1} \cdots i_{k} \| j} F^{i_{1} \cdots i_{k} j \| 0} \\
& +\frac{(-)^{k} k^{2}(k+1) !}{2(D-k-1)} \pi_{i_{1} \cdots i_{k-1}}^{\prime} \pi^{\prime i_{1} \cdots i_{k-1}} .
\end{aligned}
$$

The next step - consistency of the primary constraints is solved in two stages. Initially, by direct computation one infers the Abelian character of the set of primary constraints consisting in (18) and (50)(51). This allow us to conclude that the consistency of the primary constraints reduces only to the calculations between canonical Hamiltonian and primary constraints. By direct computations one obtains

$$
\begin{aligned}
{\left[G_{i_{1} \cdots i_{k-1}}^{(1)}, H_{0}^{(I I)}\right] } & =\partial^{j} \pi_{j i_{1} \cdots i_{k-1} \| 0} \equiv G_{i_{1} \cdots i_{k-1}}^{(2)} \approx 0, \\
{\left[G_{i_{1} \cdots i_{k-1} \| j}^{(1)}, H_{0}^{(I I)}\right] } & =\partial^{l} \pi_{l i_{1} \cdots i_{k-1} \| j} \equiv G_{i_{1} \cdots i_{k-1} \| j}^{(2)} \approx 0, \\
{\left[\bar{\gamma}_{i_{1} \cdots i_{k}}^{(1)}, H_{0}^{(I I)}\right] } & =\frac{2 k+1}{2(k+1)} \partial^{j} \bar{\gamma}_{i_{1} \cdots i_{k} j}^{(1)}-G_{\left[i_{1} \cdots i_{k-1} \| i_{k}\right]}^{(2)} \approx 0, \\
{\left[\bar{\gamma}_{i_{1} \cdots i_{k+1}}^{(1)}, H_{0}^{(I I)}\right] } & =0 .
\end{aligned}
$$

The results (53)-(56) display the secondary constraints

$$
G_{i_{1} \cdots i_{k-1}}^{(2)} \approx 0, \quad G_{i_{1} \cdots i_{k-1} \| j}^{(2)} \approx 0
$$


that together with (18) and (50)-(51) constitute an Abelian set of constraints. These outputs, supplemented with the Poisson brackets

$$
\left[G_{i_{1} \cdots i_{k-1}}^{(2)}, H_{0}^{(I I)}\right]=0=\left[G_{i_{1} \cdots i_{k-1} \| j}^{(2)}, H_{0}^{(I I)}\right]
$$

allow us to conclude that the Dirac algoritm stops at this stage.

In the next, in order to count the degrees of freedom, we use: the first-class constraints (18), (50)-(51) and (57), the irreducible character of the constraints (18), (50)-(51) and the $L=k-1$ reducibilities of the secondary constraints (57)

$$
\begin{aligned}
\left(Z_{j_{1} \cdots j_{k-2}}\right)^{i_{1} \cdots i_{k-1}} G_{i_{1} \cdots i_{k-1}}^{(2)} & =0, \\
\left(Z_{l_{1} \cdots l_{k-p-3}}\right)^{j_{1} \cdots j_{k-p-2}}\left(Z_{j_{1} \cdots j_{k-p-2}}\right)^{i_{1} \cdots i_{k-p-1}} & =0, \quad p=\overline{0, k-3}, \\
\left(Z_{j_{1} \cdots j_{k-2}}\right)^{i_{1} \cdots i_{k-1} \| i} G_{i_{1} \cdots i_{k-1} \| i}^{(2)} & =0, \\
\left(Z_{l_{1} \cdots l_{k-p-3} \| l}\right)^{j_{1} \cdots i_{k-p-2} \| j}\left(Z_{j_{1} \cdots j_{k-p-2} \| j}\right)^{i_{1} \cdots i_{k-p-1} \| i} & =0, \quad p=\overline{0, k-3} .
\end{aligned}
$$

In the above, we used the notations

$$
\begin{aligned}
\left(Z_{j_{1} \cdots j_{k-p-2}}\right)^{i_{1} \cdots i_{k-1-p}} & =\partial^{\left[i_{1}\right.} \delta_{j_{1}}^{i_{2}} \cdots \delta_{j_{k-p-1}}^{\left.i_{k-p}\right]}, \quad p=\overline{0, k-2}, \\
\left(Z_{j_{1} \cdots j_{k-p-2} \| j}\right)^{i_{1} \cdots i_{k-1-p} \| i} & =\delta_{j}^{i}\left(Z_{j_{1} \cdots j_{k-p-2}}\right)^{i_{1} \cdots i_{k-1-p}}, \quad p=\overline{0, k-2} .
\end{aligned}
$$

Putting these together we get the number of independent degrees of freedom for the model under study

$$
N_{D O F}^{(I I)}=D\left(\begin{array}{c}
D-2 \\
k
\end{array}\right)-\left(\begin{array}{c}
D \\
k+1
\end{array}\right) .
$$

As in the previous situation, we are interested about the 'nature' of the degrees o freedom. In order to do this, firstly we chose the set of the canonical gauge conditions corresponding to the first-class constraints (18), (50)-(51) and (57) consisting in (42) and

$$
\begin{aligned}
\bar{\chi}^{(1) i_{1} \cdots i_{k}} & \equiv A^{i_{1} \cdots i_{k} \| 0} \approx 0, \\
\bar{\chi}^{(1) i_{1} \cdots i_{k+1}} & \equiv A^{\left[i_{1} \cdots i_{k} \| i_{k+1}\right]} \approx 0, \\
\bar{\chi}_{i_{1} \cdots i_{k-1}}^{(1)} & \equiv \pi_{i_{1} \cdots i_{k-1}}^{\prime} \approx 0, \\
\bar{\chi}^{(2) i_{1} \cdots i_{k-1}} & \equiv \partial_{i} A^{i i_{1} \cdots i_{k-1} \| 0} \approx 0, \\
\bar{\chi}^{(2) i_{1} \cdots i_{k-1} \| j} & \equiv \partial_{i} A^{i i_{1} \cdots i_{k-1} \| j} \approx 0 .
\end{aligned}
$$

Evaluating now the restriction of the first-class Hamiltonian (52) to the reduced phase space we get

$$
\begin{aligned}
\overline{\mathcal{H}}_{0}^{(I I)} \approx & -\frac{(-)^{k} k(k+1) !}{2} \pi_{i_{1} \cdots i_{k} \| \mu} \pi^{i_{1} \cdots i_{k} \| \mu}+\frac{(-)^{k}}{2(k+1)^{2}(k+1) !}\left(F_{i_{1} \cdots i_{k+1} \| j}\right)^{2} \\
& +\frac{(-)^{k}}{2 k(k+1)(k+1) !} F_{i_{1} \cdots i_{k} j \| l} F^{i_{1} \cdots i_{k} l \| j},
\end{aligned}
$$

we conclude that also in this case ghost modes do not appear.

Finally, if we pass again to the Lagrangian formulation [via extended action], we derive for the functional

$$
S_{0}^{(I I)}\left[A_{\mu_{1} \cdots \mu_{k} \| \alpha}\right]=\int \mathrm{d}^{D} x \mathcal{L}_{0}^{(I I)}
$$

the generating set of gauge transformations

$$
\delta_{\epsilon, \xi}^{(I I)} A_{\mu_{1} \cdots \mu_{k} \| \alpha}=\epsilon_{\mu_{1} \cdots \mu_{k} \alpha}+\partial_{\left[\mu_{1}\right.} \epsilon_{\left.\mu_{2} \cdots \mu_{k}\right] \alpha}-(-)^{k} k \partial_{\alpha} \epsilon_{\mu_{1} \cdots \mu_{k}}+\partial_{\left[\mu_{1}\right.} \xi_{\left.\mu_{2} \cdots \mu_{k}\right] \mid \alpha},
$$

where the gauge parameters $\epsilon_{\mu_{1} \cdots \mu_{k+1}}$ and $\epsilon_{\mu_{1} \cdots \mu_{k}}$ are completely antisymmetric

$$
\epsilon_{\mu_{1} \cdots \mu_{k+1}}=\frac{1}{(k+1) !} \epsilon_{\left[\mu_{1} \cdots \mu_{k+1}\right]}, \quad \epsilon_{\mu_{1} \cdots \mu_{k}}=\frac{1}{k !} \epsilon_{\left[\mu_{1} \cdots \mu_{k}\right]}
$$

while $\xi_{\mu_{1} \cdots \mu_{k-1} \mid \alpha}$ display the mixed symmetry $(k-1,1)$

$$
\xi_{\mu_{1} \cdots \mu_{k-1} \mid \alpha}=\frac{1}{(k-1) !} \xi_{\left[\mu_{1} \cdots \mu_{k-1}\right] \mid \alpha}, \quad \xi_{\left[\mu_{1} \cdots \mu_{k-1} \mid \alpha\right]}=0 .
$$

From (73) we infer that the irreducible component $B_{\mu_{1} \cdots \mu_{k+1}}$ is a pure gauge field

$$
\delta_{\epsilon, \xi}^{(I I)} B_{\mu_{1} \cdots \mu_{k+1}}=\epsilon_{\mu_{1} \cdots \mu_{k+1}} .
$$




\subsection{Case III}

For the choice (26) of the parameters $a_{1}$ and $a_{2}$ the Lagrangian density (6) takes the form

$$
\mathcal{L}_{0}^{(I I I)}=\frac{(-)^{k+1}}{2(k+1)(k+1) !}\left[\frac{1}{k+1}\left(F_{\mu_{1} \cdots \mu_{k+1} \| \alpha}\right)^{2}+\frac{1}{k} F_{\mu_{1} \cdots \mu_{k} \beta \| \alpha} F^{\mu_{1} \cdots \mu_{k} \alpha \| \beta}-\frac{k+1}{k(D-k)}\left(F_{\mu_{1} \cdots \mu_{k}}\right)^{2}\right] .
$$

Replacing (26) into the definitions (17) one infers the primary constraints (18) and

$$
\begin{aligned}
& \gamma_{i_{1} \cdots i_{k-1}}^{(1)} \equiv \pi_{i_{1} \cdots i_{k-1}}^{\prime} \approx 0 \\
& \bar{\gamma}_{i_{1} \cdots i_{k+1}}^{(1)} \equiv \pi_{\left[i_{1} \cdots i_{k} \| i_{k+1}\right]}+\frac{1}{k \cdot(k+1) !} F_{i_{1} \cdots i_{k+1} \| 0} \approx 0 .
\end{aligned}
$$

Moreover, solving the corresponding equations (17) in respect with some of the generalized velocities, we derive the canonical Hamiltonian density [well-defined only on the primary constraints surface]

$$
\begin{aligned}
\mathcal{H}_{0}^{(I I I)}= & -k A^{0 i_{1} \cdots i_{k-1} \| \mu}\left(\partial^{l} \pi_{l i_{1} \cdots i_{k-1} \| \mu}\right)+\frac{(-)^{k}}{2(k+1)^{2}(k+1) !}\left(F_{i_{1} \cdots i_{k+1} \| \mu}\right)^{2} \\
& -(-)^{k} \frac{k(k+1) !}{2}\left(\frac{D-k}{D-k-1}\left(\pi_{i_{1} \cdots i_{k} \| 0}\right)^{2}+\left(\pi_{i_{1} \cdots i_{k} \| j}\right)^{2}\right) \\
& -\frac{(-)^{k}}{2 k(D-k-1)(k+1) !}\left(F_{i_{1} \cdots i_{k}}^{\prime}\right)^{2}+\frac{(-)^{k}}{2 k(k+1)(k+1) !} F^{i_{1} \cdots i_{k} j \| l} F_{i_{1} \cdots i_{k} l \| j} \\
& +\frac{(-)^{k}}{D-k-1} \pi_{i_{1} \cdots i_{k} \| 0} F^{\prime i_{1} \cdots i_{k}}-\frac{(-)^{k}}{2(k+1)} \pi_{i_{1} \cdots i_{k} \| j} F^{i_{1} \cdots i_{k} j \| 0} .
\end{aligned}
$$

As in the previous two situations, the primary constraints (18) and (78)-(79) are Abelian so their consistency reduces to the computation of the Poisson brackets between them and the canonical Hamiltonian (80). By direct calculations we infer

$$
\begin{aligned}
{\left[G_{i_{1} \cdots i_{k-1}}^{(1)}, H_{0}^{(I I I)}\right] } & =\partial^{j} \pi_{j i_{1} \cdots i_{k-1} \| 0} \equiv G_{i_{1} \cdots i_{k-1}}^{(2)} \approx 0, \\
{\left[G_{i_{1} \cdots i_{k-1} \| j}^{(1)}, H_{0}^{(I I I)}\right] } & =\partial^{l} \pi_{l i_{1} \cdots i_{k-1} \| j} \equiv G_{i_{1} \cdots i_{k-1} \| j}^{(2)} \approx 0, \\
{\left[\gamma_{i_{1} \cdots i_{k-1}}^{(1)}, H_{0}^{(I I I)}\right] } & =(-)^{k} G_{i_{1} \cdots i_{k-1}}^{(2)} \approx 0, \\
{\left[\bar{\gamma}_{i_{1} \cdots i_{k+1}}^{(1)}, H_{0}^{(I I I)}\right] } & =-(-)^{k}\left(\partial_{\left[i_{1}\right.} \pi_{\left.i_{2} \cdots i_{k+1}\right] \| 0}-\frac{(-)^{k}}{k(k+1) !} \partial^{j} F_{i_{1} \cdots i_{k+1} \| j}\right) \\
& \equiv-(-)^{k} \bar{\gamma}_{i_{1} \cdots i_{k+1}}^{(2)} \approx 0 .
\end{aligned}
$$

The results (81)- (84) put into evidence the secondary constraints

$$
G_{i_{1} \cdots i_{k-1}}^{(2)} \approx 0, \quad G_{i_{1} \cdots i_{k-1} \| j}^{(2)} \approx 0, \quad \bar{\gamma}_{i_{1} \cdots i_{k+1}}^{(2)} \approx 0
$$

Direct computations show that the Poisson brackets among the constraints (18), (78)-(79) and (85) are vanishing so that the requirement of conservation in time for the secondary constraints (85) reduces, as in the previous situation, to the computation of the Poisson brackets between (85) and canonical Hamiltonian (80)

$$
\begin{gathered}
{\left[G_{i_{1} \cdots i_{k-1}}^{(2)}, H_{0}^{(I I I)}\right]=0=\left[G_{i_{1} \cdots i_{k-1} \| j}^{(2)}, H_{0}^{(I I I)}\right]} \\
{\left[\bar{\gamma}_{i_{1} \cdots i_{k+1}}^{(2)}, H_{0}^{(I I I)}\right]=\frac{(-)^{k+1}}{k+1} \partial_{\left[i_{1}\right.} G_{\left.i_{2} \cdots i_{k} \| i_{k+1}\right]}^{(2)}+\frac{2 k+1}{2(k+1)} \partial^{j} \partial_{\left[i_{1}\right.} \bar{\gamma}_{\left.i_{2} \cdots i_{k+1}\right] j}^{(1)} \approx 0}
\end{gathered}
$$

The previous analysis allow us to conclude that Dirac algorithm stops at this stage and, moreover, the canonical Hamiltonian (80) coincides with the first-class Hamiltonian of the system.

In order to count the independent degrees of freedom for the model under study, we investigate the reducibilities of the first-class constraints set (18), (78)-(79) and (85). The concrete expressions of the

analyzed constraints evidence that: i) the constraints $\bar{\gamma}_{i_{1} \cdots i_{k+1}}^{(2)} \approx 0$ are off-shell reducible of order $(D-k-2)$ with the reducibility functions

$$
\left(Z_{j_{1} \cdots j_{k+p+1}}\right)^{i_{1} \cdots i_{k+p}}=\partial_{\left[j_{1}\right.} \delta_{j_{2}}^{i_{1}} \cdots \delta_{\left.j_{k+p+1}\right]}^{i_{k+p}}, \quad k=\overline{1, D-k-2},
$$


ii) the constraints (57) are $L=k-1$ off-shell reducible with the reducibility functions given in (63)-(64), iii) the constraints $\gamma_{i_{1} \cdots i_{k-1}}^{(1)} \approx 0$ and $G_{i_{1} \cdots i_{k-1} \| j}^{(2)} \approx 0$ are off-shell first order reducible

$$
\left(\partial^{\left[i_{1}\right.} \delta_{j_{1}}^{i_{2}} \cdots \delta_{j_{k-2}}^{\left.i_{k-1}\right]}\right) \gamma_{i_{1} \cdots i_{k-1}}^{(1)}+\left(-\delta_{j_{1}}^{\left[i_{1}\right.} \cdots \delta_{j_{k-2}}^{i_{k-2}} \sigma^{\left.i_{k-1}\right] j}\right) G_{i_{1} \cdots i_{k-1} \| j}^{(2)}=0
$$

and iv) the constraints (18) and (79) are irreducible. The previous reducibilities of the first-class constraints implies that the number of independent degrees of freedom is

$$
N_{D O F}^{(I I I)}=D\left(\begin{array}{c}
D-2 \\
k
\end{array}\right)-\left(\begin{array}{c}
D \\
k+1
\end{array}\right)+\left(\begin{array}{c}
D-2 \\
k-3
\end{array}\right) .
$$

At this stage we are interested if all the (90) independent degrees of freedom are physical. In order to aswer to this question we choose a set of canonical gauge conditions consisting in (42), (67), (69)-(70) and

$$
\begin{aligned}
& \tilde{\chi}^{(1) i_{1} \cdots i_{k-1}} \equiv A^{\left(i_{1} \cdots i_{k-1}\right.} \approx 0, \\
& \bar{\chi}^{(2) i_{1} \cdots i_{k+1}} \equiv \partial^{\left[i_{1}\right.} A^{\left.i_{2} \cdots i_{k+1}\right] \| 0} \approx 0 .
\end{aligned}
$$

Evaluating now the first-class Hamiltonian (80) in the presence of the canonical gauge conditions we obtain

$$
\begin{aligned}
\overline{\mathcal{H}}_{0}^{(I I I)} \approx & \frac{k(k+1) !}{2}\left(\pi_{i_{1} \cdots i_{k} \| j}^{2}-\frac{D-k}{D-k-1} \pi_{i_{1} \cdots i_{k} \| 0}^{2}\right) \\
& +\frac{(-)^{k}}{2(k+1)^{2}(k+1) !}\left(F_{i_{1} \cdots i_{k+1} \| j}\right)^{2}+\frac{(-)^{k}}{2 k(k+1)(k+1) !} F^{i_{1} \cdots i_{k} j \| l} F_{i_{1} \cdots i_{k} l \| j} .
\end{aligned}
$$

Analyzing now the expression (93) we observe that the modes $\pi_{i_{1} \cdots i_{k} \| 0}$ comes with negative contributions in the kinetic term so we conclude that ghost modes are present in this situation.

Finally, the returning to the Lagrangian formulation [via the extended action] furnishes for the functional

$$
S_{0}^{(I I I)}\left[A_{\mu_{1} \cdots \mu_{k} \| \alpha}\right]=\int \mathrm{d}^{D} x \mathcal{L}_{0}^{(I I I)}
$$

the generating set of gauge transformations

$$
\delta_{\epsilon, \xi}^{(I I I)} A_{\mu_{1} \cdots \mu_{k} \| \alpha}=\sigma_{\alpha\left[\mu_{1}\right.} \epsilon_{\left.\mu_{2} \cdots \mu_{k}\right]}+\partial^{\sigma} \epsilon_{\mu_{1} \cdots \mu_{k} \alpha \sigma}+\partial_{\left[\mu_{1}\right.} \epsilon_{\left.\mu_{2} \cdots \mu_{k}\right] \alpha}+\partial_{\left[\mu_{1}\right.} \xi_{\left.\mu_{2} \cdots \mu_{k}\right] \mid \alpha},
$$

where the gauge parameters of $\epsilon$-type are completely antisymmetric and those of $\xi$-type possess the mixed symmetry $(k-1,1)$. It is easy to see, that in the present situation, the gauge transformations of the irreducible components become

$$
\begin{aligned}
\delta_{\epsilon, \xi}^{(I I I)} t_{\mu_{1} \cdots \mu_{k} \mid \alpha}= & \sigma_{\alpha\left[\mu_{1}\right.} \epsilon_{\left.\mu_{2} \cdots \mu_{k}\right]}+\frac{1}{k+1}\left(\partial_{\left[\mu_{1}\right.} \epsilon_{\left.\mu_{2} \cdots \mu_{k}\right] \alpha}-(-)^{k} k \partial_{\alpha} \epsilon_{\mu_{1} \cdots \mu_{k}}\right) \\
& +\partial_{\left[\mu_{1}\right.} \xi_{\left.\mu_{2} \cdots \mu_{k}\right] \mid \alpha}, \\
\delta_{\epsilon, \xi}^{(I I I)} B_{\mu_{1} \cdots \mu_{k} \alpha}= & \partial^{\sigma} \epsilon_{\mu_{1} \cdots \mu_{k} \alpha \sigma}+\frac{k}{k+1} \partial_{\left[\mu_{1}\right.} \epsilon_{\left.\mu_{2} \cdots \mu_{k} \alpha\right]} .
\end{aligned}
$$

It is woth noticing the presence in the generating set (95) of some conformal-like [first term in the righthand side of [95)] and BF-type [second component in the right-hand side of (95)] gauge transformations.

\subsection{Case IV}

From the dynamical point of view, this situation is quite similar to the previous one as we shall see in the following. Making the choice (27) in the local function (6), the Lagrangian density becomes

$$
\mathcal{L}_{0}^{(I V)} \equiv \frac{(-)^{k+1}}{2(k+1)(k+1) !}\left[\frac{1}{k+1}\left(F_{\mu_{1} \cdots \mu_{k+1} \| \alpha}\right)^{2}+\frac{1}{k} F_{\mu_{1} \cdots \mu_{k} \beta \| \alpha} F^{\mu_{1} \cdots \mu_{k} \alpha \| \beta}-(-)^{k} a\left(F_{\mu_{1} \cdots \mu_{k}}\right)^{2}\right],
$$

where $a$ is an arbitrary real constant with the range given in (27). 
In this context, the definitions (17) of the canonical momenta lead to the Abelian primary constraints (18) and (79) and also produce the canonical Hamiltonian density [well defined only on the primary constraint surface]

$$
\begin{aligned}
\mathcal{H}_{0}^{(I V)=} & -k A^{0 i_{1} \cdots i_{k-1} \| \mu}\left(\partial^{l} \pi_{l i_{1} \cdots i_{k-1} \| \mu}\right)+\frac{(-)^{k}}{2(k+1)^{2}(k+1) !}\left(F_{i_{1} \cdots i_{k+1} \| \mu}\right)^{2} \\
& -(-)^{k} \frac{k(k+1) !}{2}\left(\frac{k+1}{k+1-(-)^{k} k a}\left(\pi_{i_{1} \cdots i_{k} \| 0}\right)^{2}+\left(\pi_{i_{1} \cdots i_{k} \| j}\right)^{2}\right) \\
& -\frac{a k^{3}(k+1) !}{2\left[k+1-(-)^{k} a k(D-k)\right]}\left(\pi_{i_{1} \cdots i_{k-1}}^{\prime}\right)^{2}+\frac{k a}{k+1-(-)^{k} k a} \pi_{i_{1} \cdots i_{k} \| 0} F^{i_{1} \cdots i_{k}} \\
& -\frac{a}{2\left(k+1-(-)^{k} k a\right)(k+1) !}\left(F_{i_{1} \cdots i_{k}}^{\prime}\right)^{2}+\frac{(-)^{k}}{2 k(k+1)(k+1) !} F^{i_{1} \cdots i_{k} j \| l} F_{i_{1} \cdots i_{k} l \| j} \\
& -\frac{(-)^{k}}{2(k+1)} \pi_{i_{1} \cdots i_{k} \| j} F^{i_{1} \cdots i_{k} j \| 0} .
\end{aligned}
$$

The second step in the Dirac analysis — consistency of the primary constraints — involves the computations between the canonical Hamiltonian and the primary constraints. Due to the fact that the primary constraints are Abelian, their consistency requirement produces the secondary constraints (85) as

$$
\left[G_{i_{1} \cdots i_{k-1}}^{(1)}, H_{0}^{(I V)}\right] \equiv G_{i_{1} \cdots i_{k-1}}^{(2)}, \quad\left[G_{i_{1} \cdots i_{k-1} \| j}^{(1)}, H_{0}^{(I V)}\right] \equiv G_{i_{1} \cdots i_{k-1} \| j}^{(2)}, \quad\left[\bar{\gamma}_{i_{1} \cdots i_{k+1}}^{(1)}, H_{0}^{(I V)}\right] \equiv-(-)^{k} \bar{\gamma}_{i_{1} \cdots i_{k+1}}^{(2)} .
$$

Concerning the consistency of the secondary constraints (85) this does not imply new constraints because firstly, the constraints (18), (79) and (85) are Abelian and secondly, the Poisson brackets between the canonical Hamiltonian (99) and secondary constraints (85) reads as in (86)- 877).

The previous results allow us to state that the Dirac algorithm stops at this level, and, moreover, (99) is nothing but the first-class Hamiltonian.

In order to count the independent degrees of freedom, we use the reducibilities of the first-class constraints (18), (79) and (85) established in the previous situation. More precisely, the constraints: (18) and (79) are irreducible; $G_{i_{1} \cdots i_{k-1}}^{(2)} \approx 0$ and $G_{i_{1} \cdots i_{k-1} \| j}^{(2)} \approx 0$ are off-shell reducible of order $L=k-1$ with the reducibility functions given in (63)-(64) and $\bar{\gamma}_{i_{1} \cdots i_{k+1}}^{(2)} \approx 0$ are off-shell reducible of order $(D-k-2)$ with the reducibility functions expressed in (88). In view of these, the number of independent degrees of freedom in the present situation reads as

$$
N_{D O F}^{(I V)}=(D-1)\left(\begin{array}{c}
D-2 \\
k
\end{array}\right)-\left(\begin{array}{c}
D-1 \\
k+1
\end{array}\right) .
$$

As in the preceding situation we are interested whether all the degrees of freedom are physical. In order to answer the this question, we evaluate the first-class Hamiltonian (99) on the reduced phase-space. To do so, we choose the set of canonical gauge-fixing conditions consisting in (42), (67), (69), (170) and (92) and the time-evolution generator corresponding to (99) takes the form

$$
\begin{aligned}
\overline{\mathcal{H}}_{0}^{(I V) \approx} & -\frac{k(k+1)(k+1) !}{2\left(k+1-(-)^{k} k a\right)} \pi_{i_{1} \cdots i_{k} \| 0}^{2}+\frac{k(k+1) !}{2} \Pi_{i_{1} \cdots i_{k} \| j}^{2} \\
& -\frac{a^{2} k^{3}(D-k)(k+1) !}{8\left(k+1-(-)^{k} a k(D-k)\right)^{2}} \pi_{i_{1} \cdots i_{k-1}}^{2} \\
& -\frac{a}{2\left(k+1-(-)^{k} k a\right)(k+1) !}\left(F_{i_{1} \cdots i_{k}}^{\prime}\right)^{2} \\
& +\frac{(-)^{k}}{2(k+1)^{2}(k+1) !} F^{i_{1} \cdots i_{k+1} \| j}\left(F_{i_{1} \cdots i_{k+1} \| j}+\frac{(-)^{k}}{k} F_{j\left[i_{1} \cdots i_{k} \| i_{k+1}\right]}\right)
\end{aligned}
$$

where we employed the notations

$$
\Pi_{i_{1} \cdots i_{k} \| j} \equiv \pi_{i_{1} \cdots i_{k} \| j}+\frac{(-)^{k} k}{2\left(k+1-(-)^{k} a k(D-k)\right)} \pi_{\left[i_{1} \cdots i_{k-1}\right.}^{\prime} \sigma_{\left.i_{k}\right] j} .
$$

Based on the expression (102) we conclude that the unphysical degrees of freedom [ghost modes] are still present.

Invoking again the Dirac's conjecture, we derive for the functional

$$
S_{0}^{(I V)}\left[A_{\mu_{1} \cdots \mu_{k} \| \alpha}\right]=\int \mathrm{d}^{D} x \mathcal{L}_{0}^{(I V)}
$$


the generating set of gauge transformations

$$
\delta_{\epsilon, \xi}^{(I V)} A_{\mu_{1} \cdots \mu_{k} \| \alpha}=\partial^{\sigma} \epsilon_{\mu_{1} \cdots \mu_{k} \alpha \sigma}+\partial_{\left[\mu_{1}\right.} \epsilon_{\left.\mu_{2} \cdots \mu_{k}\right] \alpha}+\partial_{\left[\mu_{1}\right.} \xi_{\left.\mu_{2} \cdots \mu_{k}\right] \mid \alpha},
$$

where the gauge parameters of $\epsilon$ and $\xi$-type have the symmetries specified in the previous situation.

It is remarkable the presence in the generating set (104) of a BF-type [first term in the right-hand side of (104)] gauge component.

\subsection{Case V}

Now, the constants that parametrize (6) are taken as in (28). With this choice, the Lagrangian density (6) becomes

$$
\mathcal{L}_{0}^{(V)}=\frac{(-)^{k+1}}{2(k+1)(k+1) !}\left[\frac{1}{k+1}\left(F_{\mu_{1} \cdots \mu_{k+1} \| \alpha}\right)^{2}-(-)^{k} \bar{a} F_{\mu_{1} \cdots \mu_{k} \beta \| \alpha} F^{\mu_{1} \cdots \mu_{k} \alpha \| \beta}+\frac{(-)^{k} \bar{a}-1}{D-k}\left(F_{\mu_{1} \cdots \mu_{k}}\right)^{2}\right],
$$

where the range of the real constant $\bar{a}$ is given in (28). In this context, the definitions of the canonical momenta (17) furnish the primary constraints (18) and (78) and also produce the canonical Hamiltonian density

$$
\begin{aligned}
\mathcal{H}_{0}^{(V)=} & -k A^{0 i_{1} \cdots i_{k-1} \| \mu}\left(\partial^{l} \pi_{l i_{1} \cdots i_{k-1} \| \mu}\right)+\frac{(-)^{k}}{2(k+1)^{2}(k+1) !}\left(F_{i_{1} \cdots i_{k+1} \| \mu}\right)^{2} \\
& +\frac{(k+1)(k+1) !}{2\left(\bar{a}-(-)^{k}\right)}\left(\frac{D-k}{D-k-1}\left(\pi_{i_{1} \cdots i_{k} \| 0}\right)^{2}+\left(\pi_{i_{1} \cdots i_{k} \| j}\right)^{2}\right. \\
& \left.-\frac{\bar{a}}{k \bar{a}+(-)^{k}} \pi_{i_{1} \cdots i_{k} \| j} \pi^{\left[i_{1} \cdots i_{k} \| j\right]}\right)+\frac{(-)^{k} \bar{a}}{k \bar{a}+(-)^{k}} \pi_{i_{1} \cdots i_{k} \| j} F^{i_{1} \cdots i_{k} j \| 0} \\
& +\frac{(-)^{k}}{D-k-1} \pi_{i_{1} \cdots i_{k} \| 0} F^{\prime i_{1} \cdots i_{k}}-\frac{\bar{a}^{2}}{2(k+1)(k+1) !\left(k \bar{a}+(-)^{k}\right)}\left(F_{i_{1} \cdots i_{k+1} \| 0}\right)^{2} \\
& +\frac{1}{2(k+1)(k+1) !}\left(\frac{\bar{a}-(-)^{k}}{D-k-1}\left(F_{i_{1} \cdots i_{k}}^{\prime}\right)^{2}-\bar{a} F^{i_{1} \cdots i_{k} j \| l} F_{i_{1} \cdots i_{k} l \| j}\right) .
\end{aligned}
$$

Due to the Abelian character of the primary constraints (18) and (78), the second step in the Dirac analysis reduces to the computation of the Poisson brackets between the canonical Hamiltonian (106) and the primary constraints (18) and (78). Direct calculations display

$$
\begin{aligned}
{\left[G_{i_{1} \cdots i_{k-1}}^{(1)}, H_{0}^{(V)}\right] } & =G_{i_{1} \cdots i_{k-1}}^{(2)}, \\
{\left[G_{i_{1} \cdots i_{k-1} \| j}^{(1)}, H_{0}^{(V)}\right] } & =G_{i_{1} \cdots i_{k-1} \| j}^{(2)}, \\
{\left[\gamma_{i_{1} \cdots i_{k-1}}^{(1)}, H_{0}^{(V)}\right] } & =(-)^{k} G_{i_{1} \cdots i_{k-1}}^{(2)},
\end{aligned}
$$

where the functions that appear in the right-hand side of (107)-(109) have the concrete expressions given in (81) and (83).

Due to the fact that the primary and secondary constraints depend only on the canonical momenta we conclude these are Abelian. Moreover, the Dirac algorithm stops at this level as the consistency requirements of the secondary constraints $G_{i_{1} \cdots i_{k-1}}^{(2)} \approx 0$ and $G_{i_{1} \cdots i_{k-1} \| j}^{(2)} \approx 0$ no longer produce tertiary constraints

$$
\left[G_{i_{1} \cdots i_{k-1}}^{(2)}, H_{0}^{(V)}\right]=0=\left[G_{i_{1} \cdots i_{k-1} \| j}^{(2)}, H_{0}^{(V)}\right] .
$$

At this stage we infer that the canonical Hamiltonian (106) is of the first-class and also we are able to count the number of independent degrees of freedom. In view of this, we invoke: i) the irreducible character of the first-class constraints (18), ii) the first-order reducibilities (89) of the constraints (78) and $G_{i_{1} \cdots i_{k-1} \| j}^{(2)}$, iii) the $L=k-1$ off-shell reducibilities of the constraints $G_{i_{1} \cdots i_{k-1}}^{(2)} \approx 0$ and $G_{i_{1} \cdots i_{k-1} \| j}^{(2)} \approx 0$ [the reducibility functions are given in (63)-(64)]. Based on these arguments, the number of independent degrees of freedom is

$$
N_{D O F}^{(V)}=D\left(\begin{array}{c}
D-2 \\
k
\end{array}\right)-\left(\begin{array}{c}
D-1 \\
k-1
\end{array}\right)+\left(\begin{array}{c}
D-1 \\
k-2
\end{array}\right) .
$$


As in the previous cases we ask for the nature of independent degrees of freedom. In order to answer to this question we select the canonical gauge conditions (42), (69), (70) and (91) and evaluate the restriction of the first-class Hamiltonian on the reduced phase-space

$$
\begin{aligned}
\overline{\mathcal{H}}_{0}^{(V) \approx} & (-)^{k} \frac{(k+1)(k+1) !}{2\left(\bar{a}-(-)^{k}\right)}\left(\frac{D-k}{D-k-1} \Pi_{i_{1} \cdots i_{k} \| 0}^{2}-\pi_{i_{1} \cdots i_{k} \| j}^{2}\right. \\
& \left.+\frac{1}{\left(k \bar{a}+(-)^{k}\right)(k+1)} \Pi_{i_{1} \cdots i_{k+1}}^{2}\right)+\frac{1}{2(k+1)(k+1) !} \frac{\bar{a}-(-)^{k}}{D-k}\left(F_{i_{1} \cdots i_{k}}^{\prime}\right)^{2} \\
& +\frac{1+(-)^{k}(k-1) \bar{a}-k \bar{a}^{2}}{2(k+1)^{2}(k+1) !\left(k \bar{a}+(-)^{k}\right)}\left(F_{i_{1} \cdots i_{k+1} \| 0}\right)^{2} \\
& +\frac{(-)^{k}}{2(k+1)^{2}(k+1) !} F^{i_{1} \cdots i_{k+1} \| \mu}\left(F_{i_{1} \cdots i_{k+1} \| j}-\bar{a} F_{j\left[i_{1} \cdots i_{k} \| i_{k+1}\right]}\right) .
\end{aligned}
$$

In the above we employed the notations

$$
\begin{aligned}
& \Pi_{i_{1} \cdots i_{k} \| 0}=\pi_{i_{1} \cdots i_{k} \| 0}+\frac{(-)^{k}\left(\bar{a}-(-)^{k}\right)}{(D-k)(k+1)(k+1) !} F_{i_{1} \cdots i_{k}}^{\prime}, \\
& \Pi_{i_{1} \cdots i_{k+1}}=\pi_{\left[i_{1} \cdots i_{k} \| i_{k+1}\right]}-\frac{\bar{a}-(-)^{k}}{(k+1)(k+1) !} F_{i_{1} \cdots i_{k+1} \| 0}
\end{aligned}
$$

Analyzing now the kinetic term of the generator of time-evolution (112) we conclude that also in this case the ghost modes are present.

Using the same method as in the previous subsections, one can deduce for the functional

$$
S_{0}^{(V)}\left[A_{\mu_{1} \cdots \mu_{k} \| \alpha}\right]=\int \mathrm{d}^{D} x \mathcal{L}_{0}^{(V)}
$$

the generating set of gauge transformations

$$
\delta_{\epsilon, \xi}^{(V)} A_{\mu_{1} \cdots \mu_{k} \| \alpha}=\sigma_{\alpha\left[\mu_{1}\right.} \epsilon_{\left.\mu_{2} \cdots \mu_{k}\right]}+\partial_{\left[\mu_{1}\right.} \epsilon_{\left.\mu_{2} \cdots \mu_{k}\right] \alpha}+\partial_{\left[\mu_{1}\right.} \xi_{\left.\mu_{2} \cdots \mu_{k}\right] \mid \alpha} .
$$

It is woth noticing the presence in the generating set (114) of a conformal-like [first term in the right-hand side of (114)] gauge transformation.

\subsection{Case VI}

Here we finish the canonical analysis when the real constants $a_{1}$ and $a_{2}$ have the values (29). For this setting, the Lagrangian density (6) becomes

$$
\mathcal{L}_{0}^{(V I)}=\frac{1}{2(k+1)(k+1) !}\left[\frac{(-)^{k+1}}{k+1}\left(F_{\mu_{1} \cdots \mu_{k+1} \| \alpha}\right)^{2}+\tilde{a} F_{\mu_{1} \cdots \mu_{k} \beta \| \alpha} F^{\mu_{1} \cdots \mu_{k} \alpha \| \beta}+\left((-)^{k}-\tilde{a}\right)\left(F_{\mu_{1} \cdots \mu_{k}}\right)^{2}\right] .
$$

With the choice (29), the definitions (17) lead to the primary constraints (18) and

$$
\tilde{\gamma}_{i_{1} \cdots i_{k}}^{(1)} \equiv \pi_{i_{1} \cdots i_{k} \| 0}+\frac{(-)^{k} \tilde{a}-1}{(k+1)(k+1) !} F_{i_{1} \cdots i_{k}}^{\prime} \approx 0 .
$$

Performing the Legendre transformation of (115) in respect with some of the generalized velocities [those that can be solved in the definitions of the canonical momenta [17)], we get the canonical Hamiltonian density [well defined only on the primary constraint surface]

$$
\begin{aligned}
\mathcal{H}_{0}^{(V I)}= & -k A^{0 i_{1} \cdots i_{k-1} \| \mu}\left(\partial^{l} \pi_{l i_{1} \cdots i_{k-1} \| \mu}\right)+\frac{(-)^{k}}{2(k+1)^{2}(k+1) !}\left(F_{i_{1} \cdots i_{k+1} \| j}\right)^{2} \\
& +\frac{(k+1)(k+1) !}{2\left(\tilde{a}-(-)^{k}\right)}\left(\left(\pi_{i_{1} \cdots i_{k} \| j}\right)^{2}-\frac{k}{D-k-1}\left(\pi_{i_{1} \cdots i_{k-1}}^{\prime}\right)^{2}\right. \\
& \left.-\frac{\tilde{a}}{k \tilde{a}+(-)^{k}} \pi_{i_{1} \cdots i_{k} \| j} \pi^{\left[i_{1} \cdots i_{k} \| j\right]}\right)+\frac{(-)^{k} \tilde{a}}{k \tilde{a}+(-)^{k}} \pi_{i_{1} \cdots i_{k} \| j} F^{i_{1} \cdots i_{k} j \| 0} \\
& +\frac{\tilde{a}-(-)^{k}}{2(k+1)(k+1) !}\left(F_{i_{1} \cdots i_{k}}^{\prime}\right)^{2}+\frac{1+(-)^{k} k \tilde{a}-(k+1) \tilde{a}^{2}}{2(k+1)^{2}(k+1) !\left(k \tilde{a}+(-)^{k}\right)}\left(F_{i_{1} \cdots i_{k+1} \| 0}\right)^{2} \\
& -\frac{\tilde{a}}{2(k+1)(k+1) !} F^{i_{1} \cdots i_{k} j \| l} F_{i_{1} \cdots i_{k} l \| j} .
\end{aligned}
$$


The second step in the canonical analysis - the time-preservation of the primary constraints - reduces to the computation of the Poisson brackets and primary constraints. This is due to the fact that the primary constraints (18) and (116) are Abelian. In this light, simple calculations lead to

$$
\left[G_{i_{1} \cdots i_{k-1}}^{(1)}, H_{0}^{(V I)}\right]=G_{i_{1} \cdots i_{k-1}}^{(2)}, \quad\left[G_{i_{1} \cdots i_{k-1} \| j}^{(1)}, H_{0}^{(V I)}\right]=G_{i_{1} \cdots i_{k-1} \| j}^{(2)}, \quad\left[\tilde{\gamma}_{i_{1} \cdots i_{k}}^{(1)}, H_{0}^{(V I)}\right]=-\tilde{\gamma}_{i_{1} \cdots i_{k}}^{(2)},
$$

where the functions in the right-hand sides are given in formulas (81), (82) and

$$
\tilde{\gamma}_{i_{1} \cdots i_{k}}^{(2)} \equiv-\partial^{j}\left(\pi_{i_{1} \cdots i_{k} \| j}+\frac{(-)^{k}-\tilde{a}}{(k+1)(k+1) !} F_{j i_{1} \cdots i_{k} \| 0}\right) .
$$

These results derived in the above allow to display the secondary constraints possessed by the model under study

$$
G_{i_{1} \cdots i_{k-1}}^{(2)} \approx 0, \quad G_{i_{1} \cdots i_{k-1} \| j}^{(2)} \approx 0, \quad \tilde{\gamma}_{i_{1} \cdots i_{k}}^{(2)} \approx 0 .
$$

At this stage we investigate the consistency of the secondary constraints. By direct computation we deduce that the all the constraints (18), (116) and (120) are are Abelian and, moreover, the Poisson brackets hold

$$
\left[G_{i_{1} \cdots i_{k-1}}^{(2)}, H_{0}^{(V I)}\right]=0, \quad\left[G_{i_{1} \cdots i_{k-1} \| j}^{(2)}, H_{0}^{(V I)}\right]=0=\left[\tilde{\gamma}_{i_{1} \cdots i_{k}}^{(2)}, H_{0}^{(V I)}\right] .
$$

Based on these arguments we conclude that the model under study possesses no tertiary constraints and, in addition, the canonical Hamiltonian (120) coincides with the first-class Hamiltonian.

In the light of the counting of independent degrees of freedom, we make use of the argumentation: i) the first-class constraints (18) and (116) are irreducible and ii) the two subsets in the secondary constraints (120) are off-shell reducible of order $L=k-1$ with the relations of reducibility given in (59)-(62) and the last two ones are off-shell reducible of order $L=k$ with the reducibility relations

$$
\begin{aligned}
\left(\tilde{Z}_{j_{1} \cdots j_{k-1}}\right)^{i_{1} \cdots i_{k-1} \| i} G_{i_{1} \cdots i_{k-1} \| i}^{(2)}+\left(\tilde{Z}_{j_{1} \cdots j_{k-1}}\right)^{i_{1} \cdots i_{k}} \tilde{\gamma}_{i_{1} \cdots i_{k}}^{(2)} & =0 \\
\left(\tilde{Z}_{l_{1} \cdots l_{k-2}}\right)^{j_{1} \cdots j_{k-1}}\left(\tilde{Z}_{j_{1} \cdots j_{k-1}}\right)^{i_{1} \cdots i_{k-1} \| i} & =0 \\
\left(\tilde{Z}_{l_{1} \cdots l_{k-2}}\right)^{j_{1} \cdots j_{k-1}}\left(\tilde{Z}_{j_{1} \cdots j_{k-1}}\right)^{i_{1} \cdots i_{k}} & =0 \\
\left(\tilde{Z}_{l_{1} \cdots l_{k-p-3}}\right)^{j_{1} \cdots j_{k-p-2}}\left(\tilde{Z}_{j_{1} \cdots j_{k-p-2}}\right)^{i_{1} \cdots i_{k-p-1}} & =0 \quad p=\overline{0, k-3}
\end{aligned}
$$

In the above, we used the notations

$$
\begin{aligned}
\left(\tilde{Z}_{j_{1} \cdots j_{k-1}}\right)^{i_{1} \cdots i_{k-1} \| i} & =k \partial^{[i} \delta_{j_{1}}^{i_{1}} \cdots \delta_{j_{k-1}}^{\left.i_{k-1}\right]}, \quad\left(\tilde{Z}_{j_{1} \cdots j_{k-1}}\right)^{i_{1} \cdots i_{k}}=\partial^{\left[i_{1}\right.} \delta_{j_{1}}^{i_{1}} \cdots \delta_{j_{k-1}}^{\left.i_{k}\right]} \\
\left(\tilde{Z}_{j_{1} \cdots j_{k-p-1}}\right)^{i_{1} \cdots i_{k-p}} & =\partial^{\left[i_{1}\right.} \delta_{j_{1}}^{i_{2}} \cdots \delta_{j_{k-p-1}}^{\left.i_{k-p}\right]}, \quad p=\overline{1, k-1} .
\end{aligned}
$$

Putting together the previous results we determine the number of independent degrees of freedom

$$
N_{D O F}^{(V I)}=(D-1)\left(\begin{array}{c}
D-2 \\
k
\end{array}\right)-\left(\begin{array}{c}
D-1 \\
k
\end{array}\right)
$$

As in the situations previously analyzed, at this level we are interested if there are ghost modes among the independent degrees o freedom (128). In view of this, we firstly choose the canonical gauge conditions (42), (66), (68)-(70) and

$$
\tilde{\chi}^{(2) i_{1} \cdots i_{k}} \equiv \partial_{j} A^{i_{1} \cdots i_{k} \| j} \approx 0
$$

and then we investigate the kinetic term in the restriciton of the first-class Hamiltonian (117) on the reduced phase-space

$$
\begin{aligned}
\overline{\mathcal{H}}_{0}^{(V I)} \approx & \frac{(k+1)(k+1) !}{2\left(\tilde{a}-(-)^{k}\right)}(-)^{k}\left(\pi_{i_{1} \cdots i_{k} \| j}^{2}-\pi_{i_{1} \cdots i_{k} \| 0}^{2}\right) \\
& +\frac{(-)^{k}}{2(k+1)^{2}(k+1) !} F^{i_{1} \cdots i_{k+1} \| j}\left(F_{i_{1} \cdots i_{k+1} \| j}-\tilde{a} F_{j\left[i_{1} \cdots i_{k} \| i_{k+1}\right]}\right) .
\end{aligned}
$$


From the expression in the above we conclude that also in this case ghost-modes are involved.

Employing the same procedure as in the previous subsections, we derive for the functional

$$
S_{0}^{(V I)}\left[A_{\mu_{1} \cdots \mu_{k} \| \alpha}\right]=\int \mathrm{d}^{D} x \mathcal{L}_{0}^{(V I)}
$$

the generating set of gauge transformations

$$
\delta_{\epsilon, \bar{\epsilon}, \xi}^{(V I)} A_{\mu_{1} \cdots \mu_{k} \| \alpha}=\partial_{\left[\mu_{1}\right.} \epsilon_{\left.\mu_{2} \cdots \mu_{k}\right] \alpha}+\partial_{\left[\mu_{1}\right.} \xi_{\left.\mu_{2} \cdots \mu_{k}\right] \mid \alpha}+(-)^{k} \partial_{\alpha} \bar{\epsilon}_{\mu_{1} \cdots \mu_{k}},
$$

where the bosonic gauge parameters of $\epsilon$-type are completely antisymmetric.

Is is remarkable that also in this situation, the generating set of gauge transformations (132) is richer than the original one (2).

\subsection{Case VII}

Here, the real parameters $a_{1}$ and $a_{2}$ possess the domains of values precized in (30) and the corresponding Lagrangian density

$$
\mathcal{L}_{0}^{(V I I)}=\frac{1}{2(k+1)(k+1) !}\left[-\frac{(-)^{k}}{k+1}\left(F_{\mu_{1} \cdots \mu_{k+1} \| \alpha}\right)^{2}+a_{1} F_{\mu_{1} \cdots \mu_{k} \beta \| \alpha} F^{\mu_{1} \cdots \mu_{k} \alpha \| \beta}+a_{2}\left(F_{\mu_{1} \cdots \mu_{k}}\right)^{2}\right]
$$

coincides with the original one (6). In this case, the definitions (17) of the canonical momenta display the Abelian primary constraints (18) and lead [via the Lagrangian's Legendre transformation in respect with the generalized velocities] to the canonical Hamiltonian density

$$
\begin{aligned}
\mathcal{H}_{0}^{(V I I)=} & -k A^{0 i_{1} \cdots i_{k-1} \| \mu}\left(\partial^{l} \pi_{l i_{1} \cdots i_{k-1} \| \mu}\right)+\frac{(-)^{k}}{2(k+1)^{2}(k+1) !}\left(F_{i_{1} \cdots i_{k+1} \| j}\right)^{2} \\
& +\frac{(k+1)(k+1) !}{2\left(a_{1}-(-)^{k}\right)} \pi^{i_{1} \cdots i_{k} \| j}\left(\pi_{i_{1} \cdots i_{k} \| j}-\frac{a_{1}}{k a_{1}+(-)^{k}} \pi_{\left[i_{1} \cdots i_{k} \| j\right]}\right. \\
& \left.-\frac{a_{2}}{a_{1}+a_{2}(D-k)-(-)^{k}} \pi_{\left[i_{1} \cdots i_{k-1}\right.}^{\prime} \sigma_{i_{k] j}}\right) \\
& +\frac{(k+1)(k+1) !}{2\left(a_{1}+a_{2}-(-)^{k}\right)}\left(\pi_{i_{1} \cdots i_{k} \| 0}\right)^{2}+\frac{(-)^{k} a_{1}}{k a_{1}+(-)^{k}} \pi_{i_{1} \cdots i_{k} \| j} F^{i_{1} \cdots i_{k} j \| 0} \\
& -\frac{(-)^{k} a_{2}}{a_{1}+a_{2}-(-)^{k}} F_{i_{1} \cdots i_{k}}^{\prime}\left(\pi^{i_{1} \cdots i_{k} \| 0}+\frac{(-)^{k} a_{1}-1}{2(k+1)(k+1) !} F^{i_{1} \cdots i_{k}}\right) \\
& +\frac{1+(-)^{k} k a_{1}-(k+1) a_{1}^{2}}{2(k+1)^{2}(k+1) !\left(k a_{1}+(-)^{k}\right)}\left(F_{i_{1} \cdots i_{k+1} \| 0}\right)^{2}-\frac{a_{1}}{2(k+1)(k+1) !} F^{i_{1} \cdots i_{k} j \| l} F_{i_{1} \cdots i_{k} l \| j} .
\end{aligned}
$$

At this stage, if we use the first-class character of the primary constraints (18), then, by asking them the time preservation, we get

$$
\left[G_{i_{1} \cdots i_{k-1}}^{(1)}, H_{0}^{(V I I)}\right]=G_{i_{1} \cdots i_{k-1}}^{(2)} \approx 0, \quad\left[G_{i_{1} \cdots i_{k-1} \| j}^{(1)}, H_{0}^{(V I I)}\right]=G_{i_{1} \cdots i_{k-1} \| j}^{(2)} \approx 0 .
$$

results that display the secondary constraints $G_{i_{1} \cdots i_{k-1}}^{(2)}$ and $G_{i_{1} \cdots i_{k-1} \| j}^{(2)}$ [whose concrete expressions are respectively written in (81) and (82)] . The dependence of the functions (18), (81) and (82) only on the canonical momenta lead to their Abelian character in the Poisson brackets. In this light, the consistency of the secondary constraints reduces to the computations of the Poisson brackets between them and the canonical Haniltonian. It can be checked that

$$
\left[G_{i_{1} \cdots i_{k-1}}^{(2)}, H_{0}^{(V I I)}\right]=0=\left[G_{i_{1} \cdots i_{k-1} \| j}^{(2)}, H_{0}^{(V I I)}\right]
$$

so the Dirac algorithm stops at this level.

Putting the results (135) and (136), we conclude that the canonical Hamiltonian (134) is just the firstclass Hamiltonian of the system. In view of counting the number of independent degrees of freedom, we inovke the irreducible character of the first-class constraints (18) supplemented with the $L=k-1$ reducibility functions (63) - (64) of the secondary first-class constraints $G_{i_{1} \cdots i_{k-1} \| j}^{(2)}$ and $G_{i_{1} \cdots i_{k-1} \| j}^{(2)}$ and get

$$
N_{D O F}^{(V I I)}=D\left(\begin{array}{c}
D-2 \\
k
\end{array}\right) \text {. }
$$


As in the other six situations, we are interested whether all the independent degrees of freedom are physical. In view of this, we take the canonical gauge conditions (42), (69) and (70) and then we evaluate the restriction of the firs-class Hamiltonian on the cooresponding reduced phase-space. Simple calculations reveal

$$
\begin{aligned}
\overline{\mathcal{H}}_{0}^{(V I I)} \approx & \frac{(k+1)(k+1) !}{2\left(1-(-)^{k} a_{1}\right)}\left(\pi_{i_{1} \cdots i_{k} \| j}^{2}-\frac{k a_{2}}{a_{1}+a_{2}(D-k)-(-)^{k}} \pi_{i_{1} \cdots i_{k-1}}^{\prime 2}\right. \\
& \left.-\frac{a_{1}}{(k+1)\left(k a_{1}+(-)^{k}\right)} p_{i_{1} \cdots i_{k+1}}^{2}-\frac{a_{1}-(-)^{k}}{a_{1}+a_{2}-(-)^{k}} p_{i_{1} \cdots i_{k}}^{2}\right) \\
& -\frac{a_{2}}{2(k+1)(k+1) !}\left(F_{i_{1} \cdots i_{k}}^{\prime}\right)^{2}+\frac{1+(-)^{k}(k-1) a_{1}-k a_{1}^{2}}{2(k+1)^{2}(k+1) !\left(k a_{1}+(-)^{k}\right)}\left(F_{i_{1} \cdots i_{k+1} \| 0}\right)^{2} \\
& +\frac{(-)^{k}}{2(k+1)^{2}(k+1) !} F^{i_{1} \cdots i_{k+1} \| j}\left(F_{i_{1} \cdots i_{k+1} \| j}-a_{1} F_{j\left[i_{1} \cdots i_{k} \| i_{k+1}\right]}\right),
\end{aligned}
$$

where we employed the notations

$$
\begin{aligned}
p_{i_{1} \cdots i_{k+1}} & \equiv \pi_{\left[i_{1} \cdots i_{k} \| i_{k+1}\right]}+\frac{1-(-)^{k} a_{1}}{(k+1)(k+1) !} F_{i_{1} \cdots i_{k+1} \| 0}, \\
p_{i_{1} \cdots i_{k}} & \equiv \pi_{i_{1} \cdots i_{k} \| 0}-\frac{(-)^{k} a_{2}}{(k+1)(k+1) !} F_{i_{1} \cdots i_{k}}^{\prime} .
\end{aligned}
$$

At this stage we can state that ghost modes are absent from (138) iff the real constants $a_{1}$ and $a_{2}$ are subjects of the inqualities

$$
\frac{a_{2}}{a_{1}+a_{2}(D-k)-(-)^{k}}<0, \quad \frac{a_{1}}{k a_{1}+(-)^{k}}<0, \quad \frac{a_{1}-(-)^{k}}{a_{1}+a_{2}-(-)^{k}}<0 .
$$

By analyzing the inequalities (141) we establish their incompatibility, result that imeadiately imply the ghost modes are also present in this situation.

Finally, if we return to the Lagrangian formulation [via extended action], we derive for the functional

$$
S_{0}^{(V I I)}\left[A_{\mu_{1} \cdots \mu_{k} \| \alpha}\right]=\int \mathrm{d}^{D} x \mathcal{L}_{0}^{(V I I)}
$$

the initial generating set of gauge transformations (2).

\section{First-order formulations}

In the present part we will derive the first-order formulations associated with the second-order models previously investigated. These constructions can be done using some auxiliary matter/gauge fields. More precisely, we shall show that: i) if the second-order Lagrangian can be written as a bilinear combination of some classical observables that are linearly in the field-strengths $F_{\mu_{1} \cdots \mu_{k+1} \| \alpha}$ then the corresponding firstorder formulation requires only auxiliary matter fields; ii) if the second-order Lagrangian cannot be written in terms of the classical observables that are linearly in the field-strengths $F_{\mu_{1} \cdots \mu_{k+1} \| \alpha}$ then the associated first-order Lagrangian involves some auxiliary gauge fields.

The program for constructing the first-order Lagrangian $\overline{\mathcal{L}}_{0}$ corresponding to the generic second-order one

$$
\mathcal{L}_{0}=\mathcal{L}_{0}\left(\left[A_{\mu_{1} \cdots \mu_{k} \| \alpha}\right]\right)=\frac{1}{2} F_{\mu_{1} \cdots \mu_{k+1} \| \alpha} M_{\nu_{1} \cdots \nu_{k+1} \| \beta}^{\mu_{1} \cdots \mu_{k+1} \| \alpha} F^{\nu_{1} \cdots \nu_{k+1} \| \beta},
$$

where $M_{\nu_{1} \cdots \nu_{k+1} \| \beta}^{\mu_{1} \cdots \mu_{k+1} \| \alpha}$ is a Lorentz nonderivative constant tensor, consists in the following steps:

i) one postulates for $\overline{\mathcal{L}}_{0}$ the gauge transformations

$$
\bar{\delta}_{\epsilon, \xi} A_{\mu_{1} \cdots \mu_{k} \| \alpha} \equiv \delta_{\epsilon, \xi} A_{\mu_{1} \cdots \mu_{k} \| \alpha}
$$

where $\delta A_{\mu_{1} \cdots \mu_{k} \| \alpha}$ is the generating set of gauge transformations corresponding to $\mathcal{L}_{0}$.

ii) one computes the gauge variations of the field-strengths $F_{\mu_{1} \cdots \mu_{k+1} \| \alpha}$ under (144) and looks for linear, nonderivative combinations $\mathcal{F}_{\mu_{1} \cdots \mu_{k+1} \alpha}$ of $F_{\mu_{1} \cdots \mu_{k+1} \| \alpha}$ that are invariant under (144). 
iii) if there are the tensors $\mathcal{F}_{\mu_{1} \cdots \mu_{k+1} \alpha}$ such that the Lagrangian density (143) can be written only in terms of them, then the first-order Lagrangian $\overline{\mathcal{L}}_{0}$ is obtained with the help of some bosonic matter fields $\mathcal{B}_{\mu_{1} \cdots \mu_{k+1} \alpha}$ [that display the symmetry properties of $\mathcal{F}_{\mu_{1} \cdots \mu_{k+1} \alpha}$ ].

iv) if there are no such tensors, then the first-order Lagrangian $\overline{\mathcal{L}}_{0}$ can be written as a quadratic form in an auxiliary gauge field $\omega_{\alpha \| \mu_{1} \cdots \mu_{k+1}}\left[\omega_{\alpha \| \mu_{1} \cdots \mu_{k+1}}=\frac{1}{(k+1) !} \omega_{\alpha \|\left[\mu_{1} \cdots \mu_{k+1}\right]}\right]$ with the gauge transformations specified.

In the sequel we shall apply this program for each of the seven situations analyzed in the previous subsection.

\subsection{Case I}

In this situation, the Lagrangian density (31) can be written in terms of the gauge-invariant objects $F_{\left[\mu_{1} \cdots \mu_{k+1} \| \alpha\right]}$ as

$$
\mathcal{L}_{0}^{(I)} \equiv \frac{(-)^{k+1}}{2(k+1)^{2}(k+2) !}\left(F_{\left[\mu_{1} \cdots \mu_{k+1} \| \alpha\right]}\right)^{2} .
$$

Therefore, the corresponding first-order Lagrangian, $\overline{\mathcal{L}}_{0}^{(I)}$, depends on the original gauge field [through the combination $F_{\left[\mu_{1} \cdots \mu_{k+1} \| \alpha\right]}$ and the matter $(k+2)$-form $B_{\mu_{1} \cdots \mu_{k+2}}$

$$
\begin{aligned}
\overline{\mathcal{L}}_{0}^{(I)} & \equiv \overline{\mathcal{L}}_{0}^{(I)}\left(B_{\mu_{1} \cdots \mu_{k+2}}, F_{\mu_{1} \cdots \mu_{k+1} \| \alpha}\right) \\
& =B_{\mu_{1} \cdots \mu_{k+2}}\left(\frac{1}{k+1} F^{\left[\mu_{1} \cdots \mu_{k+1} \| \mu_{k+2}\right]}-(-)^{k} \frac{(k+2) !}{2} B^{\mu_{1} \cdots \mu_{k+2}}\right) .
\end{aligned}
$$

A generating set of gauge transformations for the first-order action

$$
\bar{S}_{0}^{(I)}\left[A_{\mu_{1} \cdots \mu_{k} \| \alpha}, B_{\mu_{1} \cdots \mu_{k+2}}\right]=\int \mathrm{d}^{D} x \overline{\mathcal{L}}_{0}^{(I)}
$$

consists in

$$
\bar{\delta}_{\epsilon, \xi}^{(I)} A_{\mu_{1} \cdots \mu_{k} \| \alpha}=\delta_{\epsilon, \xi}^{(I)} A_{\mu_{1} \cdots \mu_{k} \| \alpha}, \quad \bar{\delta}_{\epsilon, \xi}^{(I)} B_{\mu_{1} \cdots \mu_{k+2}}=0,
$$

where the gauge transformations $\delta_{\epsilon, \xi}^{(I)} A_{\mu_{1} \cdots \mu_{k} \| \alpha}$ have the concrete form (46).

It is easy to see that the elimination of the auxiliary variables $B_{\mu_{1} \cdots \mu_{k+2}}$ on their own field equations

$$
\frac{\delta \overline{\mathcal{L}}_{0}^{(I)}}{\delta B_{\mu_{1} \cdots \mu_{k+2}}} \equiv \frac{1}{k+1} F^{\left[\mu_{1} \cdots \mu_{k+1} \| \mu_{k+2}\right]}-(-)^{k}(k+2) ! B^{\mu_{1} \cdots \mu_{k+2}}=0
$$

leads to

$$
B_{\mu_{1} \cdots \mu_{k+2}}=\frac{(-)^{k}}{(k+1)(k+2) !} F_{\left[\mu_{1} \cdots \mu_{k+1} \| \mu_{k+2}\right]} .
$$

Inserting the solution (150) into (146), we regain the second-order formulation

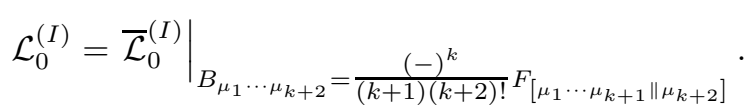

\subsection{Case II}

Following the program exposed in the beginning of this section, we firstly compute the gauge variation of the field-strength $F_{\mu_{1} \cdots \mu_{k+1} \| \alpha}$ under the gauge transformations (144) corresponding to the analyzed situation (73)

$$
\bar{\delta}_{\epsilon, \varepsilon}^{(I I)} F_{\mu_{1} \cdots \mu_{k+1} \| \alpha}=(-)^{k+1} k \partial_{\alpha}\left(\partial_{\left[\mu_{1}\right.} \epsilon_{\left.\mu_{2} \cdots \mu_{k+1}\right]}\right)+\partial_{\left[\mu_{1}\right.} \epsilon_{\left.\mu_{2} \cdots \mu_{k+1}\right] \alpha} .
$$

From the results (152) one can see that there are no combinations of $\mathcal{F}_{\mu_{1} \cdots \mu_{k+1} \alpha}$-type. In view of these, the first-order formulation associated to this limit case, also addressed in [13 for $k=1$ and $k=2$, can be done with the help of an auxiliary gauge field $\omega_{\alpha \| \mu_{1} \cdots \mu_{k+1}}\left[\omega_{\alpha \| \mu_{1} \cdots \mu_{k+1}}=\frac{1}{(k+1) !} \omega_{\alpha \|\left[\mu_{1} \cdots \mu_{k+1}\right]}\right]$ introduced 
in order to compensate the gauge variation of the field-strength $F_{\mu_{1} \cdots \mu_{k+1} \| \alpha}$ (152). We postulate for the aforementioned gauge field the gauge transformations

$$
\bar{\delta}_{\epsilon, \xi}^{(I I)} \omega_{\alpha \| \mu_{1} \cdots \mu_{k+1}}=\partial_{\alpha}\left(\epsilon_{\mu_{1} \cdots \mu_{k+1}}-k \partial_{\left[\mu_{1}\right.} \epsilon_{\left.\mu_{2} \cdots \mu_{k+1}\right]}\right)
$$

that mimic (152). At this level, one can construct the gauge-invariant tensors

$$
I_{\mu_{1} \cdots \mu_{k+1} \| \alpha} \equiv F_{\mu_{1} \cdots \mu_{k+1} \| \alpha}-\omega_{\left[\mu_{1} \| \mu_{2} \cdots \mu_{k+1}\right] \alpha},
$$

antisymmetric in the first two Lorentz indices, useful in order to write down the first-order Lagrangian density.

The first-order Lagrangian $\overline{\mathcal{L}}_{0}^{(I I)}$ is obtained subtracting from the second-order Lagrangian $\mathcal{L}_{0}^{(I I)}$ the quadratic combinations in the gauge-invariant tensors (154) that cancel the terms of $\mathcal{L}_{0}^{(I I)}$

$$
\begin{aligned}
\overline{\mathcal{L}}_{0}^{(I I)}= & \mathcal{L}_{0}^{(I I)}+\frac{(-)^{k+1}}{2(k+1)(k+1) !} I_{\mu_{1} \cdots \mu_{k+1} \| \alpha}\left(\frac{1}{k+1} I^{\mu_{1} \cdots \mu_{k+1} \| \alpha}\right. \\
& \left.+\frac{1}{k} I^{\mu_{1} \cdots \mu_{k} \alpha \| \mu_{k+1}}-\frac{1}{k} I^{\left[\mu_{1} \cdots \mu_{k}\right.} \sigma^{\left.\mu_{k+1}\right] \alpha}\right)
\end{aligned}
$$

where we used the notations

$$
I_{\mu_{1} \cdots \mu_{k}} \equiv \sigma^{\mu_{k+1} \alpha} I_{\mu_{1} \cdots \mu_{k+1} \| \alpha} .
$$

The manner just has employed for constructing the first-order Lagrangian (155) allow us to conclude that:

a) The local density (155) is gauge invariant under the gauge transformations (144) [with the right-hand side replaced by the expression [73)] and (153);

b) Eliminating in (155) the auxiliary variable on their own field equations one obtains the second-order Lagrangian (49).

Whether the first conclusion is obvious, the second one will become transparent as follows. Inserting the definitions (147) into the formula (155) one derives the concrete expression of the first-order Lagrangian density

$$
\begin{aligned}
\overline{\mathcal{L}}_{0}^{(I I) \equiv} & \overline{\mathcal{L}}_{0}^{(I I)}\left(\omega_{\alpha \| \mu_{1} \cdots \mu_{k+1}}, F_{\mu_{1} \cdots \mu_{k+1} \| \alpha}\right) \\
= & \frac{1}{k(k+1)(k+1) !} \omega^{\alpha \| \mu_{1} \cdots \mu_{k+1}}\left[\frac{1}{2}\left(\omega_{\left[\mu_{1} \| \mu_{2} \cdots \mu_{k+1}\right] \alpha}-\omega_{\left[\mu_{1} \cdots \mu_{k}\right.} \sigma_{\left.\mu_{k+1}\right] \alpha}\right)\right. \\
& \left.-F_{\mu_{1} \cdots \mu_{k+1} \| \alpha}+F_{\left[\mu_{1} \cdots \mu_{k}\right.} \sigma_{\left.\mu_{k+1}\right] \alpha}\right]
\end{aligned}
$$

where we employed the notations

$$
\omega_{\mu_{1} \cdots \mu_{k}} \equiv \sigma^{\alpha \beta} \omega_{\alpha \| \beta \mu_{1} \cdots \mu_{k}}
$$

By direct computation, one infers the field equations

$$
\begin{aligned}
\frac{\delta \overline{\mathcal{L}}_{0}^{(I I)}}{\delta \omega^{\alpha \| \mu_{1} \cdots \mu_{k+1}}} & \equiv \frac{1}{k(k+1)(k+1) !}\left[\omega_{\left[\mu_{1} \| \mu_{2} \cdots \mu_{k+1}\right] \alpha}-\omega_{\left[\mu_{1} \cdots \mu_{k}\right.} \sigma_{\left.\mu_{k+1}\right] \alpha}\right. \\
\left.-F_{\mu_{1} \cdots \mu_{k+1} \| \alpha}+F_{\left[\mu_{1} \cdots \mu_{k}\right.} \sigma_{\left.\mu_{k+1}\right] \alpha}\right] & =0
\end{aligned}
$$

whose solutions read as

$$
\omega_{\alpha \| \mu_{1} \cdots \mu_{k+1}}=(-)^{k}\left(F_{\mu_{1} \cdots \mu_{k+1} \| \alpha}-\frac{1}{k+1} F_{\left[\mu_{1} \cdots \mu_{k+1} \| \alpha\right]}\right) .
$$

Inserting the results (159) into the first-order Lagrangian density (157) we establish

$$
\mathcal{L}_{0}^{(I I)}=\left.\overline{\mathcal{L}}_{0}^{(I I)}\right|_{\omega_{\alpha \| \mu_{1} \cdots \mu_{k+1}}=(-)^{k}\left(F_{\mu_{1} \cdots \mu_{k+1} \| \alpha}-\frac{1}{k+1} F_{\left[\mu_{1} \cdots \mu_{k+1} \| \alpha\right]}\right)} .
$$




\subsection{Case III}

This case is similar to the previous one in the sense that the derivation of the first-order Lagrangian density requires some auxiliary gauge fields. This is due to the absence of the gauge-invariant $\mathcal{F}_{\mu_{1} \cdots \mu_{k+1} \alpha}$-type tensors. Precisely, if we compute the gauge variation of the field-strength $F_{\mu_{1} \cdots \mu_{k+1} \| \alpha}$ under the gauge transformations (144) corresponding to the analyzed situation (95)

$$
\bar{\delta}_{\epsilon, \varepsilon}^{(I I I)} F_{\mu_{1} \cdots \mu_{k+1} \| \alpha}=\partial_{\left[\mu_{1}\right.} \epsilon_{\mu_{2} \cdots \mu_{k}} \sigma_{\left.\mu_{k+1}\right] \alpha}+\partial^{\sigma} \partial_{\left[\mu_{1}\right.} \epsilon_{\left.\mu_{2} \cdots \mu_{k+1}\right] \alpha \sigma},
$$

we observe that we cannot identify any linearly and nonderivative combination of $F_{\mu_{1} \cdots \mu_{k+1} \| \alpha}$ that is gaugeinvariant. Nevertheless, there are some linearly and nonderivative combinations of $F_{\mu_{1} \cdots \mu_{k+1} \| \alpha}$

$$
\bar{F}_{\mu_{1} \cdots \mu_{k+1} \| \alpha} \equiv F_{\mu_{1} \cdots \mu_{k+1} \| \alpha}-\frac{1}{D-k} F_{\left[\mu_{1} \cdots \mu_{k}\right.} \sigma_{\left.\mu_{k+1}\right] \alpha},
$$

whose gauge variations do not depend on the $(k-1)$-form gauge parameter

$$
\bar{\delta}_{\epsilon, \varepsilon}^{(I I I)} \bar{F}_{\mu_{1} \cdots \mu_{k+1} \| \alpha}=\partial^{\sigma} \partial_{\left[\mu_{1}\right.} \epsilon_{\left.\mu_{2} \cdots \mu_{k+1}\right] \alpha \sigma} .
$$

The definitions (162) reveal the traceless character of the antisymmetric tensors $\bar{F}_{\mu_{1} \cdots \mu_{k+1} \| \alpha}\left[\bar{F}_{\mu_{1} \cdots \mu_{k+1} \| \alpha}=\right.$ $\frac{1}{(k+1) !} \bar{F}_{\left.\left[\mu_{1} \cdots \mu_{k+1}\right] \| \alpha\right]}$

$$
\sigma^{\mu_{k+1} \alpha} \bar{F}_{\mu_{1} \cdots \mu_{k+1} \| \alpha}=0 .
$$

In order to construct the first-order Lagrangian density, we introduce the auxiliary gauge fields $\bar{\omega}_{\alpha \| \mu_{1} \cdots \mu_{k+1}}$ with the algebraic properties of the tensors $\bar{F}_{\mu_{1} \cdots \mu_{k+1} \| \alpha}\left[\bar{\omega}_{\alpha \| \mu_{1} \cdots \mu_{k+1}}=\frac{1}{(k+1) !} \bar{\omega}_{\alpha \|\left[\mu_{1} \cdots \mu_{k+1}\right]}, \sigma^{\alpha \mu_{1}} \bar{\omega}_{\alpha \| \mu_{1} \cdots \mu_{k+1}}=\right.$ $0]$ which are subject to the gauge transformations

$$
\bar{\delta}_{\epsilon, \varepsilon}^{(I I I)} \bar{\omega}_{\alpha \| \mu_{1} \cdots \mu_{k+1}}=\partial_{\alpha} \partial^{\sigma} \epsilon_{\mu_{1} \cdots \mu_{k+1} \sigma} .
$$

Based on the results (163), the newly introduced tensor gauge fields $\bar{\omega}_{\alpha \| \mu_{1} \cdots \mu_{k+1}}$ allows to identify the gauge-invariant Lorentz tensors

$$
\bar{I}_{\mu_{1} \cdots \mu_{k+1} \| \alpha} \equiv \bar{F}_{\mu_{1} \cdots \mu_{k+1} \| \alpha}-\bar{\omega}_{\left[\mu_{1} \| \mu_{2} \cdots \mu_{k+1}\right] \alpha}
$$

that satisfy

$$
\bar{I}_{\mu_{1} \cdots \mu_{k+1} \| \alpha}=\frac{1}{(k+1) !} \bar{I}_{\left[\mu_{1} \cdots \mu_{k+1}\right] \| \alpha}, \quad \sigma^{\mu_{k+1} \alpha} \bar{I}_{\mu_{1} \cdots \mu_{k+1} \| \alpha}=0 .
$$

Proceeding as in the previous case, the first-order Lagrangian density $\overline{\mathcal{L}}_{0}^{(I I I)}$ can be written as the sum between the second-order Lagrangian $\mathcal{L}_{0}^{(I I I)}$ and some quadratic combinations in the gauge-invariant tensors (166) that cancel the terms of $\mathcal{L}_{0}^{(I I I)}$

$$
\overline{\mathcal{L}}_{0}^{(I I I)}=\mathcal{L}_{0}^{(I I I)}+\frac{(-)^{k}}{2(k+1)(k+1) !} \bar{I}_{\mu_{1} \cdots \mu_{k+1} \| \alpha}\left(\frac{1}{k+1} \bar{I}^{\mu_{1} \cdots \mu_{k+1} \| \alpha}+\frac{1}{k} \bar{I}^{\mu_{1} \cdots \mu_{k} \alpha \| \mu_{k+1}}\right)
$$

By construction, the Lagrangian density is manifestly gauge-invariaant under the gauge transformations (144) [with the right-hand side replaced by the expression (95)] and (165). Also, this reduces to the secondorder Lagrangian (77) by eliminating the auxiliary fields $\bar{\omega}_{\alpha \| \mu_{1} \cdots \mu_{k+1}}$ on their own field equations as we shall see.

Inserting the definitions (166) into the relation (168) one obtains the concrete expression of the first-order Lagrangian density

$$
\begin{aligned}
\overline{\mathcal{L}}_{0}^{(I I I)} & \equiv \overline{\mathcal{L}}_{0}^{(I I I)}\left(\bar{\omega}_{\alpha \| \mu_{1} \cdots \mu_{k+1}}, F_{\mu_{1} \cdots \mu_{k+1} \| \alpha}\right) \\
& =\frac{1}{(k+1)(k+1) !} \bar{\omega}^{\alpha \| \mu_{1} \cdots \mu_{k+1}}\left(\frac{1}{2} \bar{\omega}_{\left[\mu_{1} \| \mu_{2} \cdots \mu_{k+1}\right] \alpha}-\bar{F}_{\mu_{1} \cdots \mu_{k+1} \| \alpha}\right) .
\end{aligned}
$$

The field equations in respect with the auxiliary variables are

$$
\frac{\delta \overline{\mathcal{L}}_{0}^{(I I I)}}{\delta \bar{\omega}^{\alpha \| \mu_{1} \cdots \mu_{k+1}}} \equiv \frac{1}{k(k+1)(k+1) !}\left(\bar{F}_{\mu_{1} \cdots \mu_{k+1} \| \alpha}-\bar{\omega}_{\left[\mu_{1} \| \mu_{2} \cdots \mu_{k+1}\right] \alpha}\right)=0
$$


whose solutions read as

$$
\bar{\omega}_{\alpha \| \mu_{1} \cdots \mu_{k+1}}=(-)^{k}\left(\bar{F}_{\mu_{1} \cdots \mu_{k+1} \| \alpha}-\frac{1}{k+1} \bar{F}_{\left[\mu_{1} \cdots \mu_{k+1} \| \alpha\right]}\right) .
$$

Inserting the results (170) into the first-order Lagrangian density (169) we establish

$$
\mathcal{L}_{0}^{(I I I)}=\left.\overline{\mathcal{L}}_{0}^{(I I I)}\right|_{\bar{\omega}_{\alpha \| \mu_{1} \cdots \mu_{k+1}}=(-)^{k}}\left(\bar{F}_{\mu_{1} \cdots \mu_{k+1} \| \alpha}-\frac{1}{k+1} \bar{F}_{\left[\mu_{1} \ldots \mu_{k+1} \| \alpha\right]}\right) .
$$

\subsection{Case IV}

The present case is, in some sense, a mixing situation of the cases analyzed in 4.1 and 4.3 Precisely, there are some gauge-invariant tensors of $\mathcal{F}_{\mu_{1} \cdots \mu_{k+1} \alpha}$-type but, however, the second-order Lagrangian density (98) cannot be written in terms of them as a bilinear combinations (143). Nevertheless, the local function (98) can be represented as the sum between the second-order Lagrangian density (77) and some non-trivial terms that are quadratic in the aforementioned gauge-invariant tensors

$$
\mathcal{L}_{0}^{(I V)}=\mathcal{L}_{0}^{(I I I)}+\frac{1}{2(k+1)(k+1) !}\left(a-(-)^{k} \frac{k+1}{k(D-k)}\right) F_{\mu_{1} \cdots \mu_{k}} F^{\mu_{1} \cdots \mu_{k}} .
$$

Each of the terms in the right-hand side of the decomposition (173) is gauge-invariant under the gauge transformations (144) associated to the case under discussion (104). Indeed, using the definitions (5) we derive the gauge variations of the field-strength $F_{\mu_{1} \cdots \mu_{k+1} \| \alpha}$ under the gauge transformations (104)

$$
\bar{\delta}_{\epsilon, \varepsilon}^{(I V)} F_{\mu_{1} \cdots \mu_{k+1} \| \alpha}=\partial^{\sigma} \partial_{\left[\mu_{1}\right.} \epsilon_{\left.\mu_{2} \cdots \mu_{k+1}\right] \alpha \sigma},
$$

that lead to the gauge-invariance of the trace $F_{\mu_{1} \cdots \mu_{k}}$

$$
\bar{\delta}_{\epsilon, \varepsilon}^{(I V)} F_{\mu_{1} \cdots \mu_{k}}=0 .
$$

The previous discussion supplemented with the results established in the subsections 4.1 and 4.3 allow us to linearize the second-order Lagrangian density (173) with the help of the auxiliary tensor fields $\bar{\omega}_{\alpha \| \mu_{1} \cdots \mu_{k+1}}$

$$
\bar{\omega}_{\alpha \| \mu_{1} \cdots \mu_{k+1}}=\frac{1}{(k+1) !} \bar{\omega}_{\alpha \|\left[\mu_{1} \cdots \mu_{k+1}\right]}, \quad \sigma^{\alpha \mu_{1}} \bar{\omega}_{\alpha \| \mu_{1} \cdots \mu_{k+1}}=0
$$

and $B_{\mu_{1} \cdots \mu_{k}}\left[B_{\mu_{1} \cdots \mu_{k}}=\frac{1}{k !} B_{\left[\mu_{1} \cdots \mu_{k}\right]}\right]$ subject to the gauge transformations

$$
\bar{\delta}_{\epsilon, \varepsilon}^{(I V)} \bar{\omega}_{\alpha \| \mu_{1} \cdots \mu_{k+1}}=\partial_{\alpha} \partial^{\sigma} \epsilon_{\mu_{1} \cdots \mu_{k+1} \sigma}, \quad \bar{\delta}_{\epsilon, \varepsilon}^{(I V)} B_{\mu_{1} \cdots \mu_{k}}=0 .
$$

The auxiliary gauge fields $\bar{\omega}_{\alpha \| \mu_{1} \cdots \mu_{k+1}}$ are responsible with the linearization of the first term in the right-hand side of the decomposition (173) while the auxiliary matter vector field $B_{\mu_{1} \cdots \mu_{k}}$ is used to build the first-order formulation associated with the second term in the right-hand side decomposition (173).

Invoking again the procedures developed in the subsections 4.1 and 4.3, we can write down the first-order Lagrangian density corresponding to the second-order one (173)

$$
\begin{aligned}
\overline{\mathcal{L}}_{0}^{(I V) \equiv} \equiv & \overline{\mathcal{L}}_{0}^{(I V)}\left(\bar{\omega}_{\alpha \| \mu_{1} \cdots \mu_{k+1}}, B_{\mu_{1} \cdots \mu_{k}}, F_{\mu_{1} \cdots \mu_{k+1} \| \alpha}\right) \\
= & \frac{1}{(k+1)(k+1) !} \bar{\omega}^{\alpha \| \mu_{1} \cdots \mu_{k+1}}\left(\frac{1}{2} \bar{\omega}_{\left[\mu_{1} \| \mu_{2} \cdots \mu_{k+1}\right] \alpha}-\bar{F}_{\mu_{1} \cdots \mu_{k+1} \| \alpha}\right) \\
& +B_{\mu_{1} \cdots \mu_{k}}\left(F^{\mu_{1} \cdots \mu_{k}}-\frac{(k+1)(k+1) !}{2\left(a-(-)^{k} \frac{k+1}{k(D-k)}\right)} B^{\mu_{1} \cdots \mu_{k}}\right)
\end{aligned}
$$

By construction, the local function (178) is manifestly gauge-invariant under the gauge transformations (104) and (177) and, moreover, the elimination of the auxiliary fields $\bar{\omega}_{\alpha \| \mu_{1} \cdots \mu_{k+1}}$ and $B_{\mu_{1} \cdots \mu_{k}}$ on their field equations reduces (178) to the second-order Lagrangian density (98). Indeed, the field equations

$$
\begin{aligned}
\frac{\delta \overline{\mathcal{L}}_{0}^{(I V)}}{\delta \bar{\omega}^{\alpha \| \mu_{1} \cdots \mu_{k+1}}} & \equiv \frac{1}{k(k+1)(k+1) !}\left(\bar{F}_{\mu_{1} \cdots \mu_{k+1} \| \alpha}-\bar{\omega}_{\left[\mu_{1} \| \mu_{2} \cdots \mu_{k+1}\right] \alpha}\right)=0, \\
\frac{\delta \overline{\mathcal{L}}_{0}^{(I V)}}{\delta B^{\mu_{1} \cdots \mu_{k}}} & \equiv F_{\mu_{1} \cdots \mu_{k}}-\frac{(k+1)(k+1) !}{a-(-)^{k} \frac{k+1}{k(D-k)}} B_{\mu_{1} \cdots \mu_{k}}=0,
\end{aligned}
$$


possess the solutions

$$
\begin{aligned}
\bar{\omega}_{\alpha \| \mu_{1} \cdots \mu_{k+1}} & =(-)^{k}\left(\bar{F}_{\mu_{1} \cdots \mu_{k+1} \| \alpha}-\frac{1}{k+1} \bar{F}_{\left[\mu_{1} \cdots \mu_{k+1} \| \alpha\right]}\right), \\
B_{\mu_{1} \cdots \mu_{k}} & =\frac{a-(-)^{k} \frac{k+1}{k(D-k)}}{(k+1)(k+1) !} F_{\mu_{1} \cdots \mu_{k}} .
\end{aligned}
$$

The Lorentz tensors $\bar{F}_{\mu_{1} \ldots \mu_{k+1} \| \alpha}$ that appear in the formulas (179) and (181) have the concrete expressions (162).

Inserting the solutions (181) - 182) into the first-order Lagrangian density (178) one finally gets

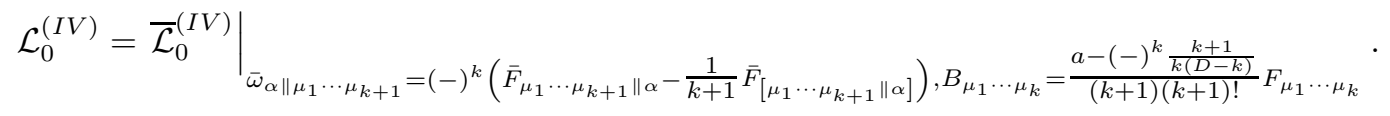

\subsection{Case V}

In this situation there are gauge-invariant tensors of $\mathcal{F}_{\mu_{1} \ldots \mu_{k+1} \alpha}$-type that allows the representation of the corresponding second-order Lagrangian density quadratically in them. Indeed, the local function (105) can be written in terms of the tensors (162) as

$$
\mathcal{L}_{0}^{(V)}=\frac{1}{2(k+1)(k+1) !} \bar{F}_{\mu_{1} \cdots \mu_{k+1} \| \alpha}\left(-\frac{(-)^{k}}{k+1} \bar{F}^{\mu_{1} \cdots \mu_{k+1} \| \alpha}+\bar{a} \bar{F}^{\mu_{1} \cdots \mu_{k} \alpha \| \mu_{k+1}}\right) .
$$

The quantities that play the role of the gauge-invariant tensors are $\bar{F}_{\mu_{1} \cdots \mu_{k+1} \| \alpha}$ as they verify

$$
\bar{\delta}_{\epsilon, \varepsilon}^{(V)} \bar{F}_{\mu_{1} \cdots \mu_{k+1} \| \alpha}=0,
$$

under the gauge transformations (144) corresponding to the analyzed situation (114).

By simple algebraic manipulations, the Lagrangian density (184) can be bringed into the form

$$
\begin{aligned}
\mathcal{L}_{0}^{(V)}= & \frac{(-)^{k+1}}{2(k+1)^{3}(k+1) !} \bar{F}_{\left[\mu_{1} \cdots \mu_{k+1} \| \alpha\right]} \bar{F}^{\left[\mu_{1} \cdots \mu_{k+1} \| \alpha\right]} \\
& +\frac{(-)^{k}\left(\bar{a}-(-)^{k}\right)}{2(k+1)^{2}(k+1) !} \bar{F}_{\mu_{1} \cdots \mu_{k+1} \| \alpha} \bar{F}^{\alpha\left[\mu_{1} \cdots \mu_{k} \| \mu_{k+1}\right]},
\end{aligned}
$$

that suggests the auxiliary matter fields needed for the linearization procedure. The first term in the righthand side of the expression (186) can be linearized with the help of a matter $(k+2)$-form with coefficients $\bar{B}_{\mu_{1} \cdots \mu_{k+2}}\left[\bar{B}_{\mu_{1} \cdots \mu_{k+2}}=\frac{1}{(k+2) !} \bar{B}_{\left[\mu_{1} \cdots \mu_{k+2}\right]}\right]$ while the second through the auxiliary matter fields $\bar{B}_{\alpha \| \mu_{1} \cdots \mu_{k+1}}$ that satisfy

$$
\bar{B}_{\alpha \| \mu_{1} \cdots \mu_{k+1}}=\frac{1}{(k+1) !} \bar{B}_{\alpha \|\left[\mu_{1} \cdots \mu_{k+1}\right]}, \quad \sigma^{\alpha \mu_{1}} \bar{B}_{\alpha \| \mu_{1} \cdots \mu_{k+1}}=0 .
$$

The first-order Lagrangian density associated with (105) reads as

$$
\begin{aligned}
\overline{\mathcal{L}}_{0}^{(V) \equiv} & \overline{\mathcal{L}}_{0}^{(V)}\left(\bar{B}_{\mu_{1} \cdots \mu_{k+2}}, \bar{B}_{\alpha \| \mu_{1} \cdots \mu_{k+1}}, F_{\mu_{1} \cdots \mu_{k+1} \| \alpha}\right) \\
= & \bar{B}_{\mu_{1} \cdots \mu_{k+2}}\left(\bar{F}^{\left[\mu_{1} \cdots \mu_{k+1} \| \mu_{k+2}\right]}+(-)^{k} \frac{(k+1)^{3}(k+1) !}{2} \bar{B}^{\mu_{1} \cdots \mu_{k+2}}\right) \\
& +\frac{(-)^{k}-\bar{a}}{(3 k+2)(k+1)(k+1) !} \bar{B}_{\alpha \| \mu_{1} \cdots \mu_{k+1}}\left(\frac{1}{2} \bar{B}^{\alpha \| \mu_{1} \cdots \mu_{k+1}}+\bar{F}^{\mu_{1} \cdots \mu_{k+1} \| \alpha}\right. \\
& \left.+(-)^{k} \frac{k+2}{2(3 k+2)} \bar{B}^{\left[\mu_{1} \| \mu_{2} \cdots \mu_{k+1}\right] \alpha}+(-)^{k} \bar{F}^{\alpha\left[\mu_{1} \cdots \mu_{k} \| \mu_{k+1}\right]}\right)
\end{aligned}
$$

and this is manifestly gauge-invariant under the gauge transformations (144) [corresponding to the analyzed situation [114)] and

$$
\bar{\delta}_{\epsilon, \varepsilon}^{(V)} \bar{B}_{\mu_{1} \cdots \mu_{k+2}}=0=\bar{\delta}_{\epsilon, \varepsilon}^{(V)} \bar{B}_{\alpha \| \mu_{1} \cdots \mu_{k+1}} .
$$

Moreover, the elimination of the auxiliary fields in (188) on their own field equations leads to the second-order Lagrangian density (105). Indeed, the field equations

$$
\begin{aligned}
& \frac{\delta \overline{\mathcal{L}}_{0}^{(V)}}{\delta \bar{B}^{\mu_{1} \cdots \mu_{k+2}}} \equiv \bar{F}_{\left[\mu_{1} \cdots \mu_{k+1} \| \mu_{k+2}\right]}+(-)^{k}(k+1)^{3}(k+1) ! \bar{B}_{\mu_{1} \cdots \mu_{k+2}}=0, \\
& \frac{\delta \overline{\mathcal{L}}_{0}^{(V)}}{\delta \bar{B}^{\alpha \| \mu_{1} \cdots \mu_{k+1}}} \equiv \frac{(-)^{k}-\bar{a}}{(3 k+2)(k+1)(k+1) !}\left(\bar{B}_{\alpha \| \mu_{1} \cdots \mu_{k+1}}+\bar{F}_{\mu_{1} \cdots \mu_{k+1} \| \alpha}\right. \\
& \left.+(-)^{k} \frac{k+2}{3 k+2} \bar{B}_{\left[\mu_{1} \| \mu_{2} \cdots \mu_{k+1}\right] \alpha}+(-)^{k} \bar{F}_{\alpha\left[\mu_{1} \cdots \mu_{k} \| \mu_{k+1}\right]}\right)=0
\end{aligned}
$$


possess the solutions

$$
\begin{aligned}
\bar{B}_{\mu_{1} \cdots \mu_{k+2}} & =\frac{(-)^{k+1}}{(k+1)^{3}(k+1) !} \bar{F}_{\left[\mu_{1} \cdots \mu_{k+1} \| \mu_{k+2}\right]}, \\
\bar{B}_{\alpha \| \mu_{1} \cdots \mu_{k+1}} & =-\frac{3 k+2}{2 k+1}\left(\bar{F}_{\mu_{1} \cdots \mu_{k+1} \| \alpha}+\frac{1}{k} \bar{F}_{\left[\mu_{1} \cdots \mu_{k+1} \| \alpha\right]}\right) .
\end{aligned}
$$

Replacing the solutions (192)-(193) into the first-order Lagrangian density (188) one finally reaches to the second-order Lagrangian density (105)

$$
\mathcal{L}_{0}^{(V)}=\left.\overline{\mathcal{L}}_{0}^{(V)}\right|_{\bar{B}=\bar{B}(\bar{F})}
$$

\subsection{Case VI}

This case mixes, in some sense, the situations analyzed in 4.1 and 4.2 This is basically due to the expression of the second-oder Lagrangian density (115), local function that can be rewritten under the form

$$
\mathcal{L}_{0}^{(V I)} \equiv \frac{1+(-)^{k} k \tilde{a}}{k+1} \mathcal{L}_{0}^{(I)}\left(F_{\mu_{1} \cdots \mu_{k+1} \| \alpha}\right)+\frac{\left(1-(-)^{k} \tilde{a}\right) k}{k+1} \mathcal{L}_{0}^{(I I)}\left(F_{\mu_{1} \cdots \mu_{k+1} \| \alpha}\right) .
$$

The decomposition in the above supplemented with the results obtained in the subsections 4.1 and 4.2 allow to linearize the second-oder Lagrangian density (115) with the help of two sets of auxiliary fields. First of them, denoted by $B_{\mu_{1} \cdots \mu_{k+2}}$, is a matter $(k+2)$-form $\left[B_{\mu_{1} \cdots \mu_{k+2}}=\frac{1}{(k+2) !} B_{\left[\mu_{1} \cdots \mu_{k+2}\right]}\right]$

$$
\bar{\delta}_{\epsilon, \xi}^{(V I)} B_{\mu_{1} \cdots \mu_{k+2}}=0
$$

responsible with the linearization of the first term in the right-hand side of the decomposition (195). The second ones, denoted by $\tilde{\omega}_{\alpha \| \mu_{1} \cdots \mu_{k+1}}$ is a gauge field

$$
\bar{\delta}_{\epsilon, \bar{\epsilon}, \xi}^{(V I)} \tilde{\omega}_{\alpha \| \mu_{1} \cdots \mu_{k+1}}=\partial_{\alpha} \partial_{\left[\mu_{1}\right.} \bar{\epsilon}_{\left.\mu_{2} \cdots \mu_{k+1}\right]}
$$

antisymmetric in its last two Lorentz indices and is introduced in order to build the first-order density associated with the second term in the right-hand side of the decomposition (195).

Invoking again the procedures developed in the subsections 4.1 and 4.2 we can write down the first-order Lagrangian density corresponding to the second-order one (195)

$$
\begin{aligned}
\overline{\mathcal{L}}_{0}^{(V I)} & \equiv \overline{\mathcal{L}}_{0}^{(V I)}\left(B_{\mu_{1} \cdots \mu_{k+2}}, \tilde{\omega}_{\alpha \| \mu_{1} \cdots \mu_{k+1}}, F_{\mu_{1} \cdots \mu_{k+1} \| \alpha}\right) \\
& \equiv \frac{1+(-)^{k} k \tilde{a}}{k+1} \overline{\mathcal{L}}_{0}^{(I)}\left(B_{\mu_{1} \cdots \mu_{k+2}}, F_{\mu_{1} \cdots \mu_{k+1} \| \alpha}\right)+\frac{\left(1-(-)^{k} \tilde{a}\right) k}{k+1} \overline{\mathcal{L}}_{0}^{(I I)}\left(\tilde{\omega}_{\alpha \| \mu_{1} \cdots \mu_{k+1}}, F_{\mu_{1} \cdots \mu_{k+1} \| \alpha}\right),
\end{aligned}
$$

where the first term in the right-hand side is given in (146) and the second one has the expression (157).

By construction, the Lagrangian density is invariant under the gauge transformations (196), (197) and

$$
\bar{\delta}_{\epsilon, \bar{\epsilon}, \xi}^{(V I)} A_{\mu_{1} \cdots \mu_{k} \| \alpha}=\partial_{\left[\mu_{1}\right.} \epsilon_{\left.\mu_{2} \cdots \mu_{k}\right] \alpha}+\partial_{\left[\mu_{1}\right.} \xi_{\left.\mu_{2} \cdots \mu_{k}\right] \mid \alpha}+(-)^{k} \partial_{\alpha} \bar{\epsilon}_{\mu_{1} \cdots \mu_{k}} .
$$

The field equations corresponding to the auxiliary variables

$$
\frac{\delta \overline{\mathcal{L}}_{0}^{(V I)}}{\delta B_{\mu_{1} \cdots \mu_{k+2}}}=0, \quad \frac{\delta \overline{\mathcal{L}}_{0}^{(V I)}}{\delta \tilde{\omega}^{\alpha \| \mu_{1} \cdots \mu_{k+1}}}=0,
$$

have the solutions given in (150) and (159). Inserting the aforementioned solutions into the first-order Lagrangian density (198) one reaches the initial second-order Lagrangian density (195). 


\subsection{Case VII}

In this last case, the linearization procedure requires only matter fields. This is due to the existence of the gauge-invariant tensors of $\mathcal{F}_{\mu_{1} \cdots \mu_{k+1} \alpha}$-type that allow the representation of the second-order Lagrangian (133) as a bilinear combination in them. The aforementioned gauge-invariant quantities are exactly the components of the field-strength $F_{\mu_{1} \cdots \mu_{k+1} \| \alpha}$

$$
\bar{\delta}_{\epsilon}^{(V I I)} F_{\mu_{1} \cdots \mu_{k+1} \| \alpha}=0,
$$

where the gauge transformations are given by (144) with the right-hand side expressed by (2).

In view of constructing the first-order Lagrangian density associated with the second-order one (133) we firstly rewrite this as

$$
\begin{aligned}
\mathcal{L}_{0}^{(V I I)}= & \frac{1+(-)^{k} k a_{1}}{k+1} \mathcal{L}_{0}^{(I)}\left(F_{\mu_{1} \cdots \mu_{k+1} \| \alpha}\right) \\
& +(-)^{k} \frac{k\left(a_{1}+a_{2}(D-k)-(-)^{k}\right)}{(D-k-1)(k+1)} \mathcal{L}_{0}^{(I I)}\left(F_{\mu_{1} \cdots \mu_{k+1} \| \alpha}\right) \\
& +\frac{k(D-k)\left(1-(-)^{k}\left(a_{1}+a_{2}\right)\right)}{(D-k-1)(k+1)} \mathcal{L}_{0}^{(I I I)}\left(F_{\mu_{1} \cdots \mu_{k+1} \| \alpha}\right)
\end{aligned}
$$

and then we make use of the results inferred in the cases 4.1, 4.2 and 4.3, The local functions that appear in the right-hand side of the expression (202) have been done in (31), (49) and (77).

The first term in the right-hand side of the decomposition (202) can be linearized with the help of the matter $(k+2)$-form $B_{\mu_{1} \cdots \mu_{k+2}}$ as in the situation 4.1 the second one will be linearized through the antisymmetric matter fields $B_{\alpha \| \mu_{1} \cdots \mu_{k+1}}\left[B_{\alpha \| \mu_{1} \cdots \mu_{k+1}}=\frac{1}{(k+1) !} B_{\alpha \|\left[\mu_{1} \cdots \mu_{k+1}\right]}\right]$ and the last one with the help of the traceless matter fields $\bar{B}_{\alpha \| \mu_{1} \cdots \mu_{k+1}}$ that satisfy the algebraic properties (187). Precisely, the first-order Lagrangian density associated to the second-order one (133) reads as

$$
\begin{aligned}
\overline{\mathcal{L}}_{0}^{(V I I)} \equiv & \overline{\mathcal{L}}_{0}^{(V I I)}\left(B_{\mu \nu \rho}, B_{\mu \| \alpha \beta}, \bar{B}_{\mu \| \alpha \beta}, F_{\mu \nu \| \rho}\right) \\
= & \frac{1+(-)^{k} k a_{1}}{k+1} \overline{\mathcal{L}}_{0}^{(I)}\left(B_{\mu_{1} \cdots \mu_{k+2}}, F_{\mu_{1} \cdots \mu_{k+1} \| \alpha}\right) \\
& +(-)^{k} \frac{k\left(a_{1}+a_{2}(D-k)-(-)^{k}\right)}{(D-k-1)(k+1)} \overline{\mathcal{L}}_{0}^{(I I)}\left(B_{\alpha \| \mu_{1} \cdots \mu_{k+1}}, F_{\mu_{1} \cdots \mu_{k+1} \| \alpha}\right) \\
& +\frac{k(D-k)\left(1-(-)^{k}\left(a_{1}+a_{2}\right)\right)}{(D-k-1)(k+1)} \overline{\mathcal{L}}_{0}^{(I I I)}\left(\bar{B}_{\alpha \| \mu_{1} \cdots \mu_{k+1}}, F_{\mu_{1} \cdots \mu_{k+1} \| \alpha}\right),
\end{aligned}
$$

where the local functions $\overline{\mathcal{L}}_{0}^{(I)}, \overline{\mathcal{L}}_{0}^{(I I)}$ and $\overline{\mathcal{L}}_{0}^{(I I I)}$ are respectively given in (146), (157) and (169). It is obvious that the first-order Lagrangian density (203) is manifestly gauge invariant under the gauge transformations

$$
\bar{\delta}_{\epsilon} A_{\mu_{1} \cdots \mu_{k} \| \alpha}=\partial_{\left[\mu_{1}\right.} \epsilon_{\left.\mu_{2} \cdots \mu_{k}\right] \| \alpha}, \quad \bar{\delta}_{\epsilon}^{(V I I)} B_{\mu_{1} \cdots \mu_{k+2}}=0, \quad \bar{\delta}_{\epsilon}^{(V I I)} B_{\alpha \| \mu_{1} \cdots \mu_{k+1}}=0=\bar{\delta}_{\epsilon}^{(V I I)} \bar{B}_{\alpha \| \mu_{1} \cdots \mu_{k+1}}
$$

and reduces to the second-order Lagrangian (133) by the elimination of the auxiliary fields on theirs own field equations.

\section{Conclusions}

In this paper we have given a scheme of unification [at the free level] of a $(k+1)$-form and a massless tensor gauge field with the mixed symmetry $(k, 1)$. The procedure made use of a (1-)form-valued $k$-form [that can be interpreted in terms of a collection of $k$-forms] and a corresponding Lagrangian density that completely capture the tensor gauge fields both algebraic and dynamic. Initially, we have constructed the most general PT-invariant and second-order Lagrangian density that is invariant under the standard gauge transformations of the just mentioned $k$-forms. The local function depends on two arbitrary real constants [denoted by $a_{1}$ and $a_{2}$ ] and, for some values of the real $a$-parameters, reduces to standard Lagrangian densities corresponding to the Abelian $(k+1)$-form [5] and that of a tensor gauge field with the mixed symmetry $(k, 1)[6$. Then, we have done the canonical analysis of the starting Lagrangian theory. This has put into evidence a partition of the real parameters plane $\left(a_{1}, a_{2}\right)$ made by seven components. For six among the seven partition's components it has been shown the generating set of gauge transformations is richer 
than the original gauge transformations, including BF [10] and/or conformal-like [11 gauge transformations. Moreover, in each of the seven situations has been calculated the number of independent degrees of freedom and investigated the presence of unphysical degrees of freedom [ghost-modes]. At this stage, we have proved the ghost-modes are absent only in two of the seven partition's components namely when the Lagrangian density reduces to that of a Abelian $(k+1)$-form and respectively to that of a tensor gauge field with the mixed symmetry $(k, 1)$, outputs that generalize the previous results [12. Finally, we have constructed the

first-order formulations associated with the analyzed second-order Lagrangian theory. Here, for each of the seven partition's components, we have generated the first-order Lagrangian formulation. These have been done with the price of adding specific auxiliary gauge/matter fields that made possible the linearization.

\section{Acknowledgments}

The author is grateful to Professors Constantin Bizdadea and Solange-Odile Saliu for useful discussions and comments.

\section{References}

[1] A. Einstein, E. G. Straus, Ann. Math. 47, 731 (1946).

[2] E. Schrödinger, Space-Time Structure (Cambridge University Press, Cambridge, 1950).

[3] J. W. Moffat, Phys. Rev. D 19, 3554 (1979).

[4] E. M. Cioroianu, Int. J. Mod. Phys. A27 (2012) 1250189; Rom. J. Phys. 58 (2013) 529.

[5] M. Henneaux, B. Knaepen, C. Schomblond, Commun. Math. Phys. 186 (1997) 137

[6] X. Bekaert, N. Boulanger, Commun. Math. Phys. 245 (2004) 27; Commun. Math. Phys. 271 (2007) 723.

[7] P. A. M. Dirac, Can. J.Math. 2 (1950) 129.

[8] P. A. M. Dirac, Lectures on Quantum Mechanics (Academic Press, New York, 1967).

[9] M. Henneaux, C. Teitelboim, Quantization of Gauge Systems (Princeton University Press, Princeton, 1992).

[10] D. Birmingham, M. Blau, M. Rakowski, G. Thompson, Phys. Rept. 209 (1991) 129.

[11] E. S. Fradkin, A. A. Tseytlin, Phys. Rept. 119 (1985) 233.

[12] S. Deser, Gen. Relativ. Gravit. 1 (1970) 9

[13] Yu. M. Zinoviev, First Order Formalism for Mixed Symmetry Tensor Fields, hep-th/0304067. 